\title{
Pharmacological targeting of MYC-regulated IRE1/XBP1 pathway suppresses MYC-driven breast cancer
}

\begin{abstract}
Na Zhao, ${ }^{1,2,3}$ Jin Cao, ${ }^{1,2,3}$ Longyong Xu, ${ }^{1,2,3}$ Qianzi Tang, ${ }^{4}$ Lacey E. Dobrolecki,, ${ }^{1,2,3}$ Xiangdong Lv, ${ }^{1,2,3}$ Manisha Talukdar, ${ }^{5,6}$ Yang Lu, ${ }^{1,3}$ Xiaoran Wang, ${ }^{1,2,3}$ Dorothy Z. Hu, ${ }^{7}$ Qing Shi, ${ }^{1,2,3}$ Yu Xiang, ${ }^{8}$ Yunfei Wang, ${ }^{9}$ Xia Liu, ${ }^{1,2,3}$ Wen Bu, ${ }^{1,2}$ Yi Jiang, ${ }^{10}$ Mingzhou Li, ${ }^{4}$ Yingyun Gong, ${ }^{1,11}$ Zheng Sun, ${ }^{1,11}$ Haoqiang Ying, ${ }^{12}$ Bo Yuan, ${ }^{13}$ Xia Lin, ${ }^{14}$ Xin-Hua Feng, ${ }^{1,13,14}$ Sean M. Hartig, ${ }^{1}$ Feng Li, ${ }^{1}$ Haifa Shen, ${ }^{15}$ Yiwen Chen, ${ }^{9}$ Leng Han, ${ }^{8}$ Qingping Zeng, ${ }^{16}$ John B. Patterson, ${ }^{17}$ Benny Abraham Kaipparettu, ${ }^{3}$ Nagireddy Putluri, ${ }^{1}$ Frank Sicheri, ${ }^{5,6,18}$ Jeffrey M. Rosen, ${ }^{1,3}$ Michael T. Lewis, ${ }^{1,2,3}$ and Xi Chen ${ }^{1,2,3}$

'Department of Molecular and Cellular Biology, ${ }^{2}$ Lester and Sue Smith Breast Center, and ${ }^{3}$ Dan L. Duncan Cancer Center, Baylor College of Medicine, Houston, Texas, USA. ${ }^{4}$ Institute of Animal Genetics and Breeding, College of Animal Science and Technology, Sichuan Agricultural University, Chengdu, China. ${ }^{5}$ Lunenfeld-Tanenbaum Research Institute, Sinai Health System, Toronto, Ontario, Canada. ${ }^{6}$ Department of Molecular Genetics, University of Toronto, Toronto, Ontario, Canada. ${ }^{7}$ Harvard School of Dental Medicine, Boston, Massachusetts, USA. ${ }^{8}$ Department of Biochemistry and Molecular Biology, The University of Texas Health Science Center at Houston McGovern Medical School, Houston, Texas, USA. ${ }^{9}$ Department of Bioinformatics and Computational Biology, University of Texas MD Anderson Cancer Center, Houston, Texas, USA. ${ }^{10}$ Division of Biochemical Genetics, Baylor Genetics, Houston, Texas, USA. "Division of Diabetes, Endocrinology and Metabolism, Department of Medicine, Baylor College of Medicine, Houston, Texas, USA. ${ }^{2}$ Department of Molecular and Cellular Oncology, The University of Texas MD Anderson Cancer Center, Houston, Texas, USA. ${ }^{13}$ Life Sciences Institute and Innovation Center for Cell Signaling Network, Zhejiang University, Hangzhou, Zhejiang, China. ${ }^{14}$ Michael E. DeBakey Department of Surgery, Baylor College of Medicine, Houston, Texas, USA. ${ }^{15}$ Department of Nanomedicine, Houston Methodist Research Institute, Houston, Texas, USA. ${ }^{16}$ Fosun Orinove PharmaTech Inc., Suzhou, Jiangsu, China. ${ }^{17}$ Fosun Orinove PharmaTech Inc., Newbury Park, California, USA. ${ }^{18}$ Department of Biochemistry, University of Toronto, Toronto, Ontario, Canada.
\end{abstract}

\begin{abstract}
The unfolded protein response (UPR) is a cellular homeostatic mechanism that is activated in many human cancers and plays pivotal roles in tumor progression and therapy resistance. However, the molecular mechanisms for UPR activation and regulation in cancer cells remain elusive. Here, we show that oncogenic MYC regulates the inositol-requiring enzyme 1 (IRE1)/ X-box binding protein 1 (XBP1) branch of the UPR in breast cancer via multiple mechanisms. We found that MYC directly controls IRE1 transcription by binding to its promoter and enhancer. Furthermore, MYC forms a transcriptional complex with XBP1, a target of IRE1, and enhances its transcriptional activity. Importantly, we demonstrate that XBP1 is a synthetic lethal partner of MYC. Silencing of XBP1 selectively blocked the growth of MYC-hyperactivated cells. Pharmacological inhibition of IRE1 RNase activity with small molecule inhibitor $\mathbf{8 8 6 6}$ selectively restrained the MYC-overexpressing tumor growth in vivo in a cohort of preclinical patient-derived xenograft models and genetically engineered mouse models. Strikingly, 8866 substantially enhanced the efficacy of docetaxel chemotherapy, resulting in rapid regression of MYC-overexpressing tumors. Collectively, these data establish the synthetic lethal interaction of the IRE1/XBP1 pathway with MYC hyperactivation and provide a potential therapy for MYC-driven human breast cancers.
\end{abstract}

\section{Introduction}

During tumor initiation and progression, cancer cells undergo various forms of intra- and extracellular stresses, resulting in significant proteostasis perturbations $(1,2)$. How do cancer cells survive and proliferate in the face of these cytotoxic stresses? Recent studies have shown that the unfolded protein response (UPR) is activated in many human cancers and plays important roles in tumor initiation, progression, and therapy resistance (1-7). The mammalian UPR is a 3-pronged cellular homeostatic mechanism that protects cells against stress caused by the accumulation of unfolded or misfolded proteins in the endoplasmic reticulum (ER). The

Authorship note: NZ, JC, LX, and QT contributed equally to this work. Conflict of interest: $Q Z$ and JBP are shareholders of Fosun Orinove PharmaTech Inc. MTL is a manager in StemMed Holdings LLC and a limited partner in StemMed Ltd. Submitted: June 28, 2017; Accepted: January 16, 2018.

Reference information: / Clin Invest. 2018;128(4):1283-1299.

https://doi.org/10.1172/JCI95873.
UPR is triggered by 3 distinct transmembrane sensors in the ER membrane, namely, inositol-requiring enzyme 1 (IRE1, encoded by ERN1), protein kinase RNA-like ER kinase (PERK, encoded by EIF2AK3), and activating transcription factor 6 (ATF6) $(8,9)$. Upon activation, these sensors initiate their respective downstream arms by sending signals from the ER lumen to the cytosol and the nucleus. The bifunctional kinase-RNase IRE1 is the most ancient and conserved member of the mammalian sensory triad of the UPR (10-12). Under ER stress, IRE1 oligomerizes and transautophosphorylates to activate its cytosolic RNase domain $(13,14)$. This activated form of IRE1 then excises 26 nucleotides from the mRNA of unspliced X-box binding protein 1 (XBP1), resulting in a frame shift to produce the mature, spliced XBP1 (XBP1s) (15-17). This unique cytosolic splicing mechanism enables rapid XBP1 isoform switching in response to perturbations in ER proteostasis (8).

Manipulating UPR might be clinically useful in a variety of contexts; however, several fundamental questions must be addressed in order to rationally target the UPR and improve disease outcome. 


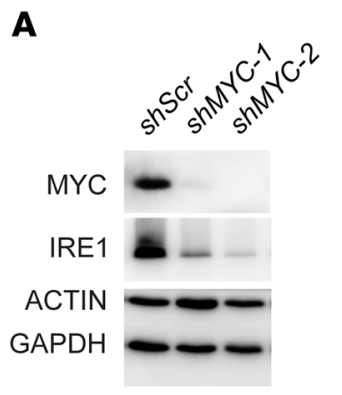

SUM159 cells

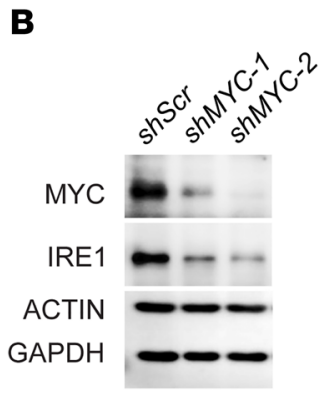

BT549 cells

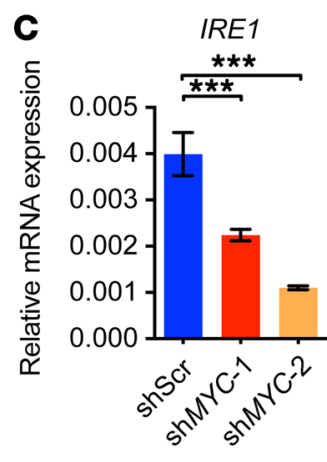

SUM159 cells

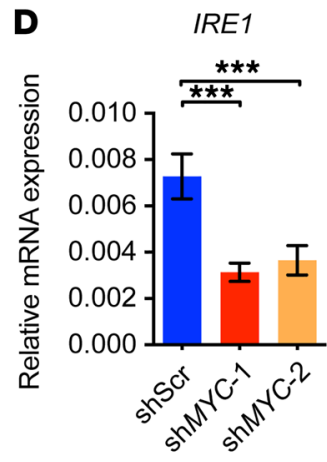

BT549 cells

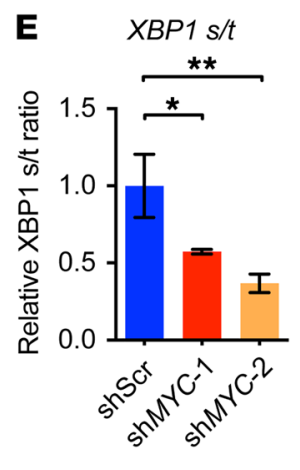

SUM159 cells

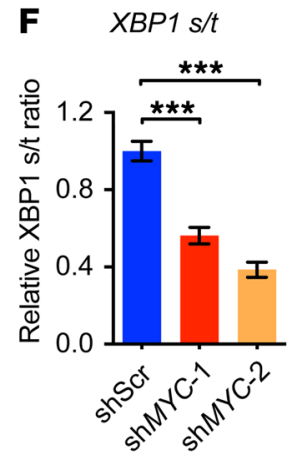

BT549 cells
Figure 1. MYC is necessary for activation of the IRE1/ XBP1 pathway. (A and B) Immunoblot of MYC and IRE1 in SUM159 cells (A) or BT549 cells (B) infected with lentiviruses encoding control scramble shRNA (shScr) or 2 distinct MYC shRNAs (shMYC-1 and shMYC-2). Actin and GAPDH were used as loading controls. (C-F) qRT-PCR analysis of IRE1 expression and XBP1 splicing in infected SUM159 cells (C and E) or BT549 cells (D and F). XBP1 s/t, the ratio of $X B P 1 s$ to total $X B P 1 t$. The $X B P 1 \mathrm{~s} / t$ ratio was normalized to that of the scramble (shScr) control. Data in QRT-PCR analysis are presented relative to actin and shown as mean $\pm \mathrm{SD}$ of technical triplicates. All data are representative of 3 independent experiments. ${ }^{*} P<0.05$; ${ }^{* *} P<0.01 ;{ }^{* *} P<0.001,1$-way ANOVA with Tukey's multiple comparison test.
Recent studies showed differential UPR activation in distinct tumor types $(1,3,4)$. However, the underlying mechanism is largely unknown. Both prolonged excessive and insufficient activation of the UPR are detrimental to cancer cells $(1,2)$; thus, the UPR must be tightly regulated for cancer cell survival. It is not clear how the UPR is regulated and integrated into oncogenic stress management in cancer cells. Though numerous compounds have been developed for targeting the UPR $(5,18-24)$, in vivo validation in patient-relevant disease models and the identification of the potential biomarkers to predict patient response are to date still lacking.

In this study, we found that oncogenic MYC directly transcriptionally activates the IRE1/XBP1 pathway by binding to the IRE1 promoter and enhancer. We show that, in the nucleus, MYC also interacts with XBP1 and enhances its transcriptional activity. Importantly, we found that MYC-hyperactivated cells are more vulnerable to $X B P 1$ inhibition and that suppression of the IRE1 RNase activity with selective small molecule inhibitor 8866 (IUPAC name: 7-hydroxy-6-methoxy-4-methyl-3-[2-(4morpholinyl)-2-oxoethyl]-2-oxo-2H-1-benzopyran-8- carboxaldehyde. CAS number: 1338934-59-0) blocks MYC-overexpressing preclinical patient-derived breast tumor and genetically engineered mouse (GEM) tumor progression and sensitizes the tumors to standard chemotherapy.

\section{Results}

MYC is necessary and sufficient for activation of the IRE1/XBP1 pathway. The IRE1/XBP1 pathway is activated in triple-negative breast cancer (TNBC) in the absence of external stimuli (3), but the underlying mechanism for this remains elusive. Since MYC expression is elevated in TNBC and has been reported as one of the key features driving TNBC $(25,26)$, we asked whether MYC is an upstream activator of IRE1/XBP1. To test this, we depleted MYC using 2 distinct shRNAs $(27,28)$ in MYC-dependent SUM159, BT549, and MDA-MB-231 breast cancer cell lines (Figure 1, A and B, and Supplemental Figure 1A; supplemental material available online with this article; https://doi.org/10.1172/JCI95873DS1). As expected, MYC knockdown decreased the expression of classical MYC targets in all 3 breast cancer cell lines (Supplemental Figure 1, B-D). Interestingly, silencing of MYC significantly reduced IRE1 at both the mRNA and protein levels in all cell lines in comparison with the scramble shRNA controls (Figure 1, A-D, and Supplemental Figure 1A). XBP1 splicing was also suppressed by MYC depletion (Figure 1, E and F, and Supplemental Figure 1A). Next, we engineered a nontransformed MCF10A human breast epithelial cell line with a tamoxifen-inducible $c$-MYC estrogen receptor fusion transgene (MCF10A ${ }^{\mathrm{MYC}-\mathrm{ER}}$ ) (Figure 2A). The treatment of MCF10A ${ }^{\text {MYC-ER }}$ cells with 4-hydroxytamoxifen (4-OHT) resulted in a dose-dependent translocation of the MYC fusion protein into the nucleus and upregulation of MYC target genes, including NCL, HSPD1, and DDX18 (Figure 2B and Supplemental Figure 2A). Notably, this MYC hyperactivation induced dose-dependent IRE1 mRNA and protein expression and XBP1 splicing (Figure 2, B and C, and Supplemental Figure 2B). Moreover, the classic XBP1 target genes DNAJB9, SEC63, HSPA5, DNAJC3, DNAJC1O, PDIA3, and $S E C 61 A 1$ were also upregulated upon MYC hyperactivation (Figure 2D and Supplemental Figure 2C). As controls, ERN2, CD59, and PMP22 were not induced by MYC (Supplemental Figure 2, C and D), suggesting that the regulation of the IRE1/XBP1 
A

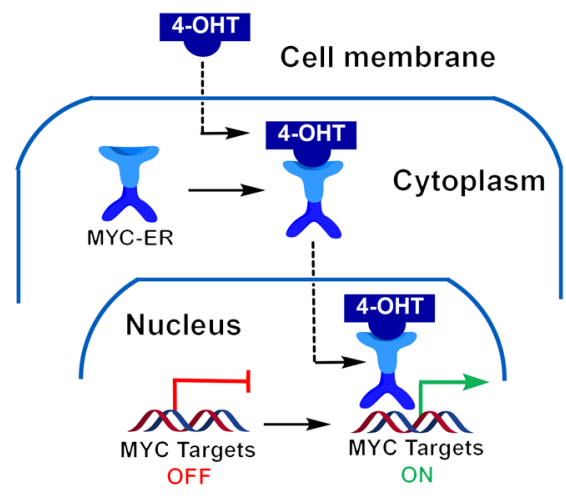

B

$\begin{array}{lllll}0 & 1 & 10 & 100 & \mathrm{nM}\end{array}$
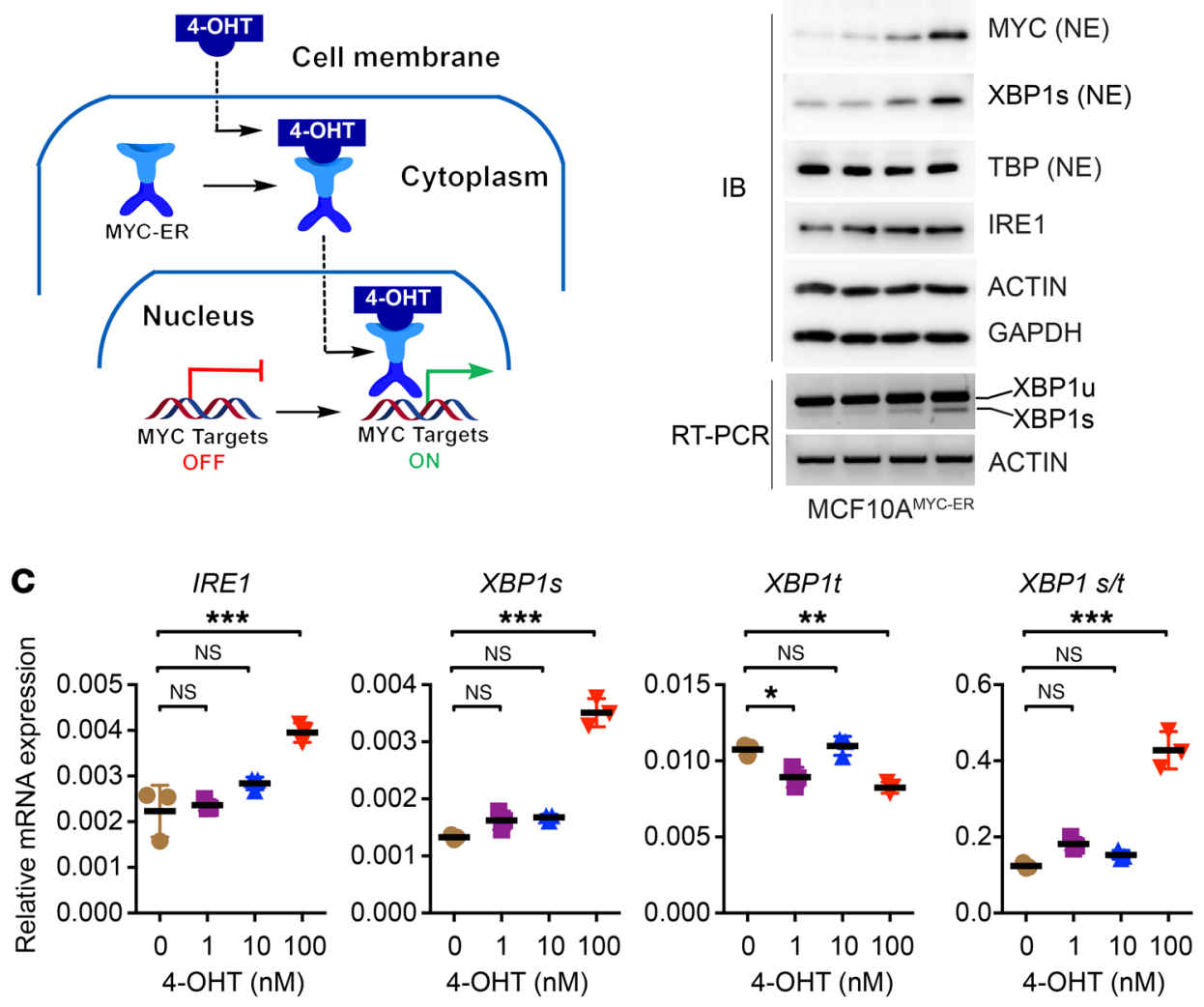

D
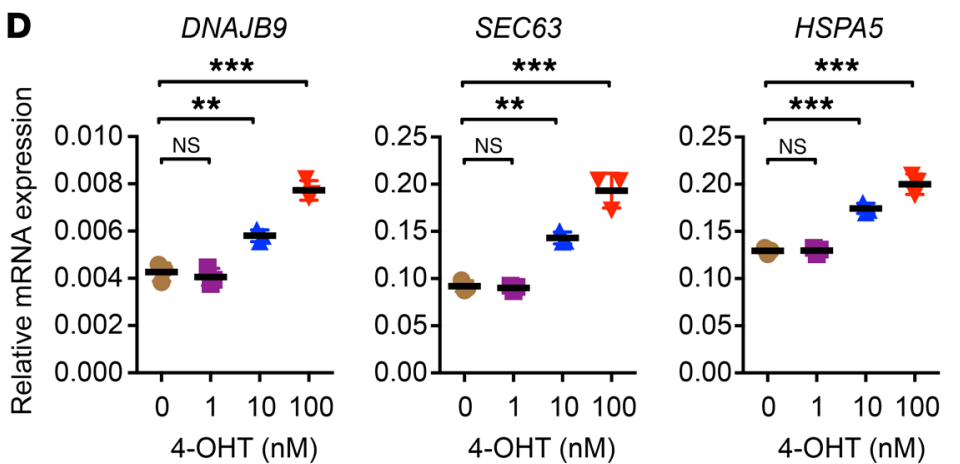

E

High

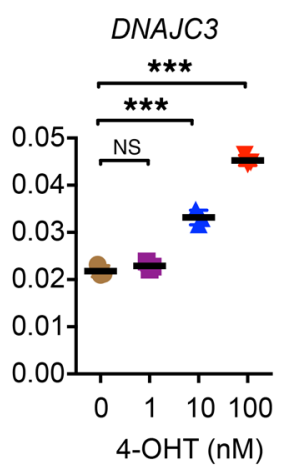

F
Medium

Low

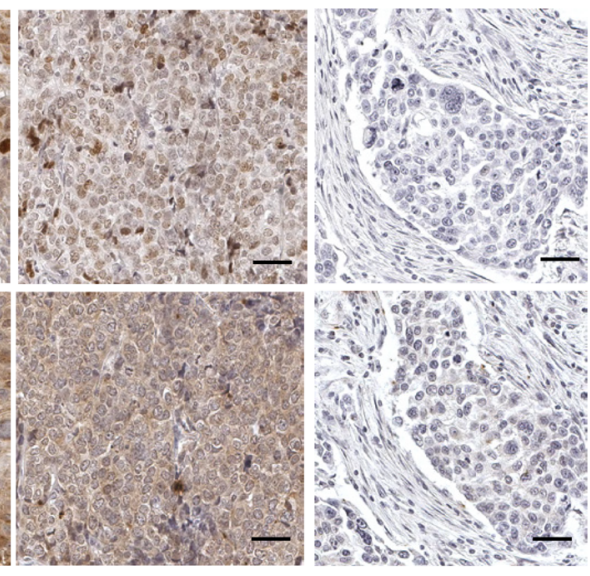

Figure 2. MYC is sufficient for activation of the IRE1/XBP1 pathway. (A)

Schematic representation of the MCF10A ${ }^{\text {MYC-ER }}$ system. In the presence of 4-OHT, MYC-ER fusion protein translocates to the nucleus and transactivates the MYC target genes. (B) Immunoblot and XBP1 splicing assay (RT-PCR) of MCF10A $^{\text {MYC-ER }}$ cells treated with different doses of 4-OHT for 24 hours. MYC-ER, XBP1s, and TBP were detected from nuclear extracts (NE) and IRE1 from whole cell lysates. TBP, actin, and CAPDH were used as loading control. (C and $\mathbf{D})$ qRT-PCR analysis of the expression of IRE1, XBP1s, XBP1 $t, X B P 1 s / t$ (C), and $X B P 1$ target genes (D) in MCF10A ${ }^{\text {MYC-ER }}$ cells treated with different doses of 4-OHT for 24 hours. ( $E$ and $\mathbf{F}$ ) The tissue microarray containing specimens from 60 breast cancer patients was subjected to IHC for MYC and IRE1 (DAB staining, brown). (E) Representative photographs are shown indicating weak, moderate, and strong staining. Scale bars: $50 \mu \mathrm{m}$. (F) MYC $\mathrm{H}$-score in tissue microarray samples with distinct IRE1 intensities. Data in qRT-PCR analysis are presented relative to actin and shown as mean \pm SD of technical triplicates. Tissue microarray was performed once, and all other data are representative of 3 independent experiments. ${ }^{*} P<0.05$; ${ }^{*} P<0.01$; ${ }^{* * *} P<0.001$, 1-way ANOVA with Tukey's multiple comparison test.
MYC

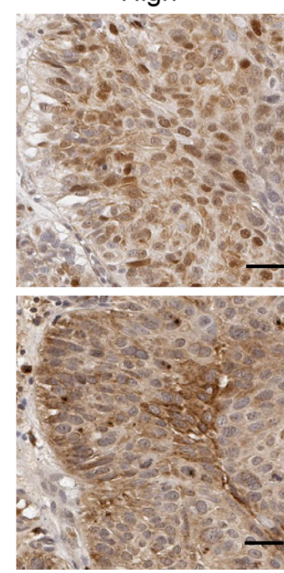

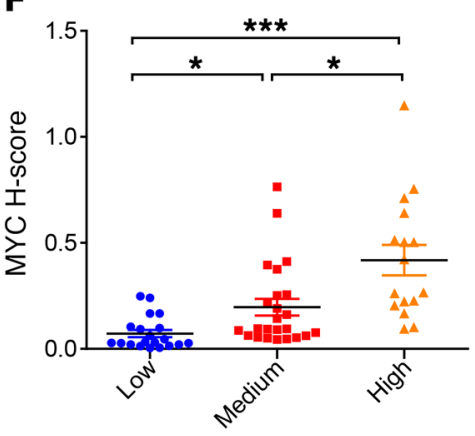

IRE1 intensity 
A

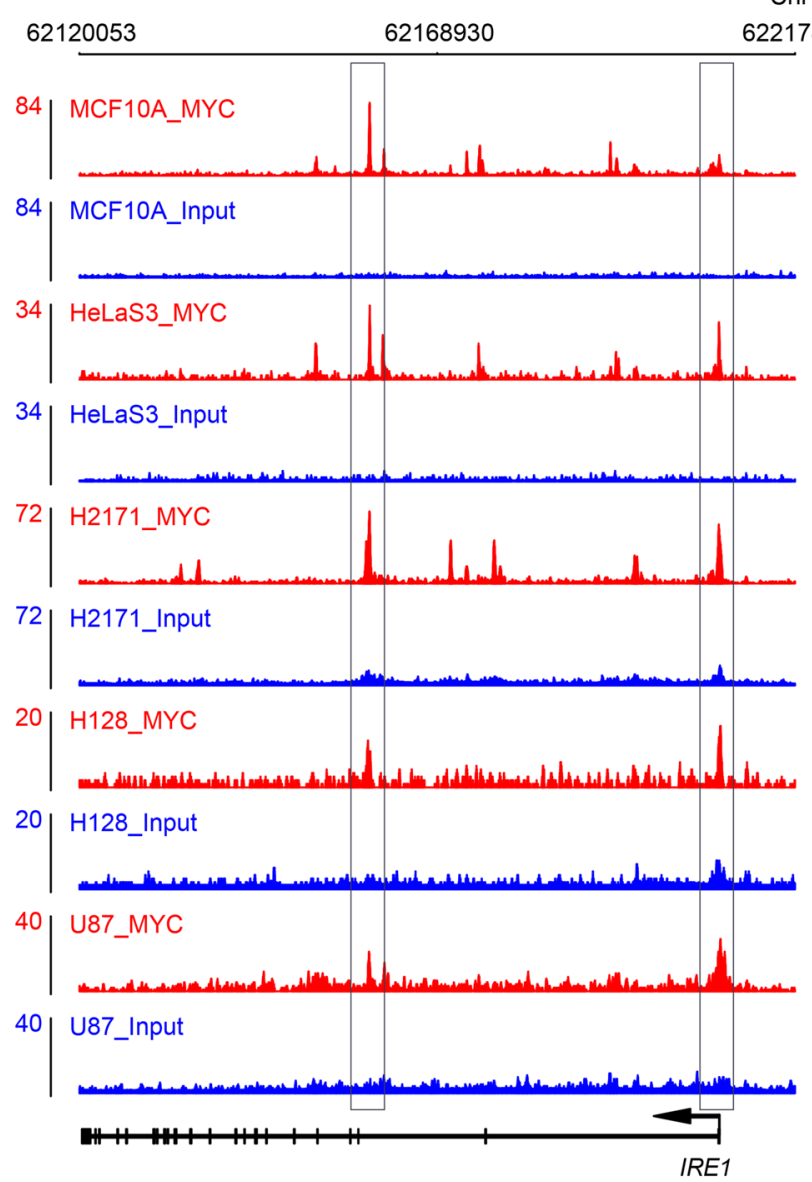

E
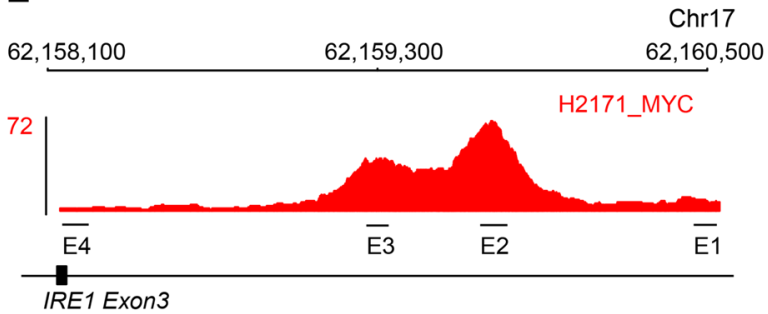

G

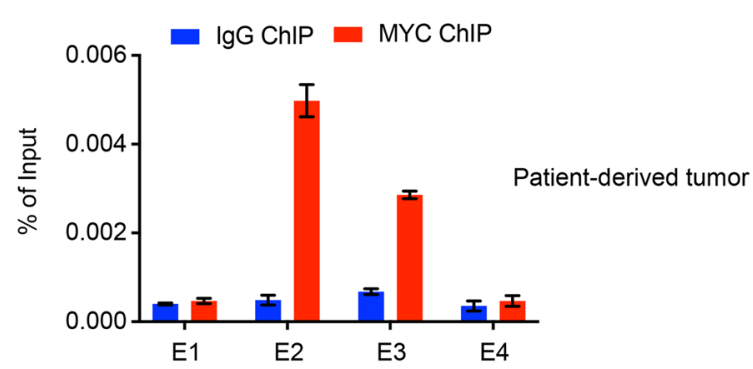

Chr17 2217807 
Figure 3. MYC binds to and regulates IRE1 proximal promoter and enhancer. (A) Track view of MYC ChIP-seq density profile on IRE1 genomic region from published data sets. (B) Schematic diagram of the ChIP primer (P1-P4) locations across the IRE1 promoter region. (C and $\mathbf{D})$ Chromatin extracts from SUM159 cells (C) and MC1 PDX tumors (D) were subjected to ChIP using anti-MYC antibody or normal IgG. Genomic regions of the IRE1 promoter were tested for enrichment of MYC binding. Data are presented relative to input and shown as mean \pm SD of technical triplicates. (E) Schematic diagram of the ChIP primer (E1-E4) locations across the IRE1 enhancer region. ( $F$ and $\mathbf{G}$ ) Chromatin extracts from SUM159 cells (F) and MC1 PDX tumors (C) were subjected to ChIP using anti-MYC antibody or normal IgG. Genomic regions of IRE1 intron were tested for enrichment of MYC binding. Data are presented relative to input and shown as mean $\pm \mathrm{SD}$ of technical triplicates. (H and I) IRE1 promoter (H) or enhancer (I) luciferase reporter was transfected into SUM159 cells infected with lentiviruses encoding scramble control shRNA (shScr) or MYC shRNA (shMYC), and luciferase activity was measured 48 hours after transfection. pGL3-basic or pGL3-promoter is the empty vector control for IRE1 promoter or enhance reporter, respectively. Data are presented relative to Renilla readings and shown as mean \pm SD of biological triplicates. All results shown are representative of 3 independent experiments. ${ }^{*} P<0.05$; ${ }^{* *} P<0.01$, 2-tailed unpaired Student's $t$ test.

pathway by MYC was not due to nonspecific global transcriptional induction. To examine the correlation between MYC and IRE1 in breast cancer patients, we performed IHC analysis of MYC and IRE1 expression in a tissue microarray composed of 60 breast cancer specimens (44 TNBC cases and 16 luminal breast cancer cases). As shown in Figure 2, E and F, IRE1 expression was highly correlated with MYC in these patients. Taken together, these data demonstrate that MYC is necessary and sufficient to activate IRE1 transcription and $X B P 1$ splicing.

$M Y C$ regulates IRE1 expression directly at the proximal promoter and enhancer. An analysis of previously published data from studies using ChIP followed by sequencing (ChIP-seq) revealed 2 MYC-binding regions on IRE1, at the proximal promoter and at an intragenic region, in multiple cell lines (29) (Figure 3A). We confirmed this by ChIP-quantitative PCR (ChIP-qPCR) experiments that showed binding of MYC to the promoter and intragenic regions of IRE1 in SUM159 cells (Figure 3, B, C, E, and F). To validate the patient relevance of the MYC binding in vivo, we performed ChIP experiments on chromatin extracted from patientderived xenograft (PDX) tumors (MC1), which express a high level of the MYC protein. As expected, in this PDX model, MYC bound to the IRE1 promoter and intragenic regions, whereas IgG showed no enrichment (Figure 3, D and G).

To test the functional importance of MYC binding, we cloned the MYC-bound IRE1 promoter and intragenic regions into the pGL3 basic and pGL3 promoter luciferase reporters, respectively. As shown in Figure 3, H and I, activities of the MYC-bound promoter and putative enhancer regions were observed in SUM159 cells, and the IRE1 promoter activity was much higher than that of the enhancer element. These activities were suppressed by the depletion of MYC by shRNA. Taken together, these data indicate that MYC controls IRE1 transcription by binding to and regulating its promoter and enhancer activities and that the IRE1 promoter is likely the major site for MYC-driven transcriptional regulation of IRE1.

$M Y C$ interacts with XBP1 and regulates XBP1 transcriptional activity. We also tested the binding of MYC to other UPR genes in SUM159 cells and patient-derived MC1 tumors, including ER- localized molecular chaperones and folding enzymes, as well as ER-associated degradation (ERAD) components that are essential to maintaining ER homeostasis and are bound and regulated by XBP1. ChIP-qPCR experiments showed that MYC and XBP1s cooccupied these UPR downstream genes (Figure 4, A-C). As a control, MYC and XBP1s did not bind to the promoters of ERN2, TSEN34, ATP5O, or NDUFA3, indicating the specificity of MYC occupancy. To further confirm their association, we performed sequential ChIP (ChIP-re-ChIP) experiments in SUM159 cells using MYC antibody followed by XBP1s antibody or control IgG antibody. Analysis of the SERP1, HSPA5, and PDIA3 promoters demonstrated that both MYC and XBP1s bind to the same DNA (Figure 4D). Reciprocal ChIP-re-ChIP experiments confirmed the association of XBP1s with MYC on the same genomic loci (Supplemental Figure 3A). These data suggest that MYC might be in the same transcriptional complex as XBP1s. Therefore, we expressed Flag-tagged MYC and HA-tagged XBP1s in 293T cells. Reciprocal coimmunoprecipitation experiments clearly demonstrated an interaction between MYC and XBP1s (Figure 4, E and F). This interaction was also observed with endogenous proteins from nuclear extracts of SUM159 and BT549 cells treated with tunicamycin (TM), a potent ER-stress inducer that blocks $N$-linked glycosylation and triggers robust $X B P 1$ splicing, for 6 hours (Figure 4, $\mathrm{G}$ and $\mathrm{H}$, and Supplemental Figure 3C). MAX is a known dimerization partner of MYC $(30,31)$ and was also found in the MYC-XBP1 complex in BT549 cells (Supplemental Figure 3, B and C). Glutathione $S$-transferase (GST) pull-down experiments further confirmed the interaction between MYC and XBP1s and showed that the central region and the transactivation domain of MYC interact with the b-zip domain of XBP1 (Supplemental Figure 3, D-I). As XBP1s and XBPu share the b-zip domain, we found XBP1u also interacted with MYC (Supplemental Figure 3, G-I). However, XBP1u is a highly unstable protein and not detectable in breast cancer cells (Supplemental Figure 3J) (3). Collectively, these data demonstrate that endogenous XBP1 interacts with MYC in the nucleus.

Next, to test the contribution of MYC to the transcriptional activity of XBP1s, we exploited 2 ER stress-response reporters, UPRE and ERSE. The cis-acting UPRE contains the preferential binding site for XBP1, while ERSE is bound by both ATF6 and XBP1 (32). Consistent with previous studies, XBP1s overexpression robustly induced UPRE- and ERSE-driven luciferase activities (Figure 4, I-L). Although MYC overexpression did not significantly transactivate UPRE and ERSE, it synergistically increased the luciferase activities of UPRE and ERSE induced by XBP1s in BT549 and 293T cells (Figure 4, I-L), suggesting that the interaction of MYC with XBP1s enhances the transcriptional activity of XBP1s. To understand how MYC promotes XBP1s transcriptional activity, we silenced MYC in SUM159 cells and examined the binding of XBP1s and coactivators (P300, SRC3, and CBP) to the MYC-XBP1 cooccupied targets. As shown in Supplemental Figure 4, A and B, MYC depletion significantly reduced XBP1 binding to DNAJC3, DNAJB9, and HSPA5 promoters, but had minimal effect on the recruitment of P300, SRC3, and CBP to the target sites. Silencing of MYC had no effect on the XBP1s protein half-life (Supplemental Figure 4C). Conversely, XBP1s did not affect MYC protein half-life either (Supplemental Figure 4D). These data indicate that MYC enhances XBP1 transcrip- 
A

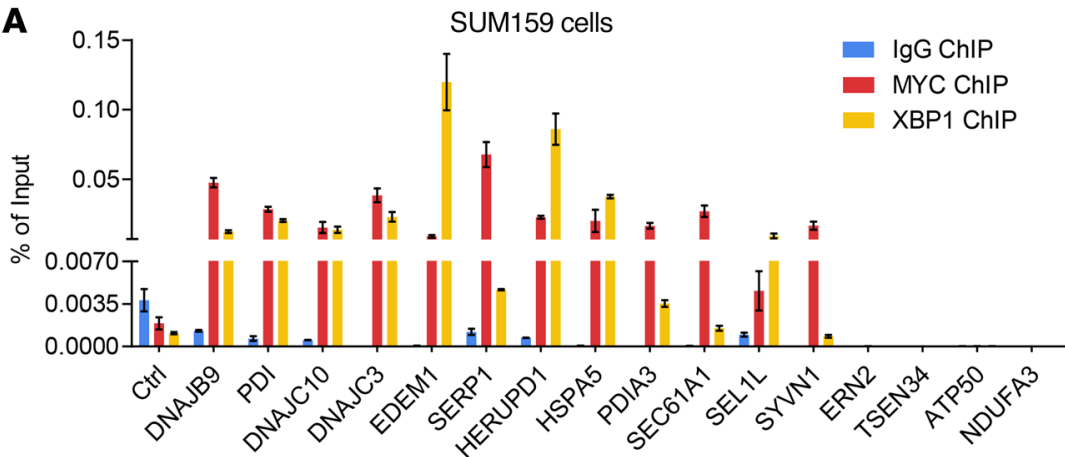

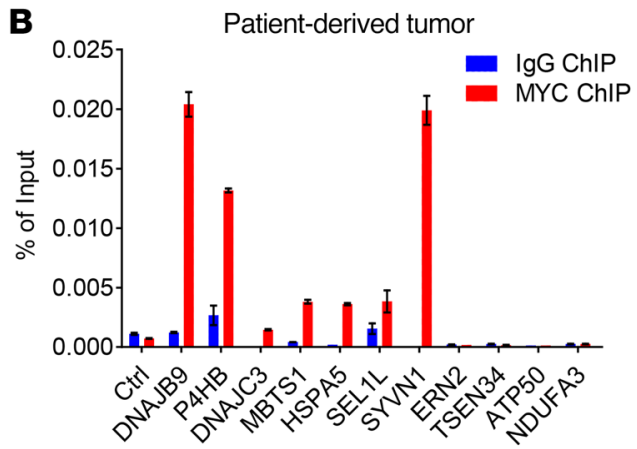

C

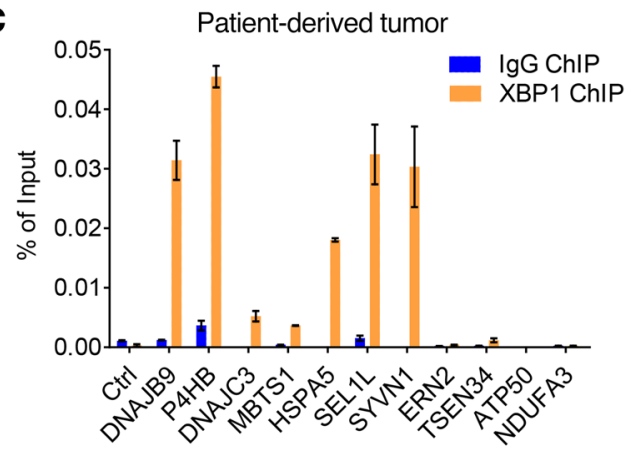

D

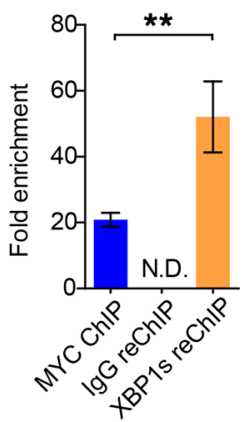

HSPA5

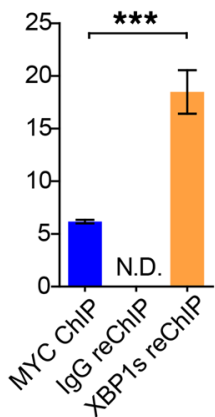

G

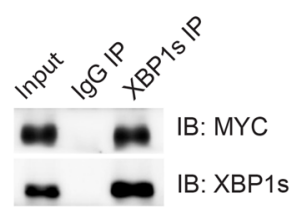

SUM159 cells
PDIA3

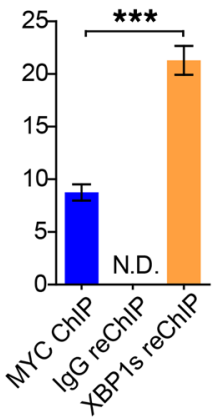

H

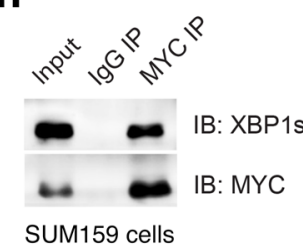

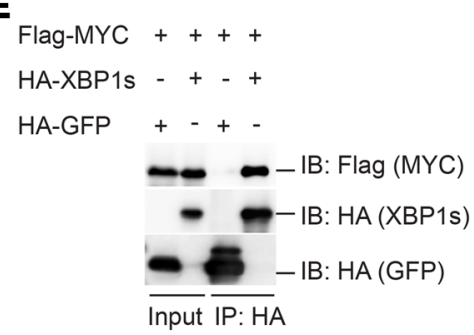

F $\mathrm{HA}-\mathrm{XBP} 1 \mathrm{~s}++++$

Flag-MYC - + - +

Flag-GFP

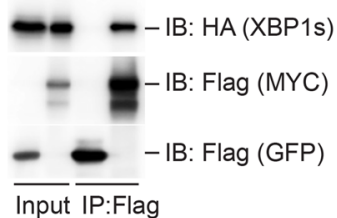

K

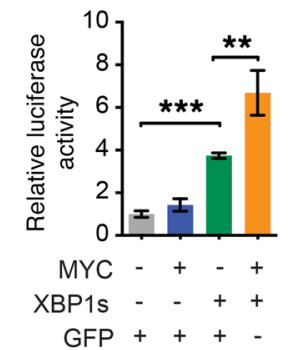

ERSE_BT549 cells

$+++$

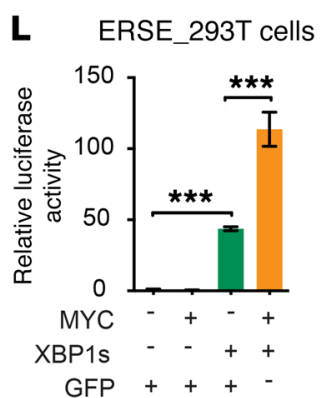

I

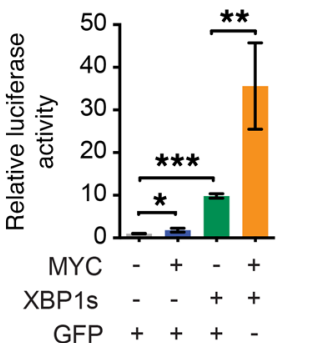

J

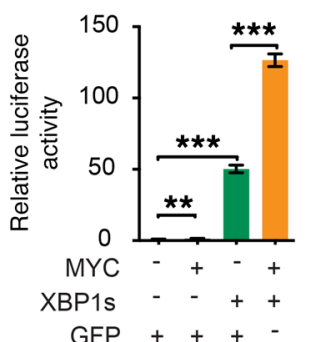

Figure 4. MYC interacts with XBP1s and regulates XBP1s transcriptional activity. (A-C) ChIP assays of SUM159 cells (A) and MC1 PDX tumors (B and C) were performed using anti-MYC or anti-XBP1s antibodies to detect enriched gene-promoter fragments. IgG was used as mock control. Genomic region upstream of VECFA lacking XBP1s- and MYC-binding sites was used as a negative control (Ctrl). Data are presented relative to input and shown as mean \pm SD of technical triplicates. (D) ChIP-re-ChIP assay of SUM159 cells was performed using the anti-MYC antibody first (MYC ChIP). Eluents were subjected to a second ChIP assay using IgG (IgG reChIP) or anti-XBP1s antibody (XBP1s reChIP) to detect enriched gene-promoter fragments. ND, not detected by qPCR assay. Data are shown as mean \pm SD of technical triplicates. (E and F) Flag-tagged MYC and HA-tagged XBP1s were coexpressed in $293 T$ cells and coimmunoprecipitation was performed with anti-HA antibody (E) or anti-Flag antibody (F). The immunoblot was probed with anti-Flag and anti-HA antibodies. HA-GFP (E) or Flag-GFP (F) was used as control. (G and $\mathbf{H}$ ) Nuclear extracts from TM-treated SUM159 cells were subjected to coimmunoprecipitation with anti-XBP1s antibody (G) or anti-MYC antibody (H). The immunoblot was probed with anti-XBP1s and anti-MYC antibodies. (I-L) UPRE or ERSE reporter was cotransfected with MYC or XBP1s or both expression plasmids into BT549 (I and K) or $293 \mathrm{~T}$ ( $\mathbf{~ a n d ~} \mathbf{L}$ ) cells. Luciferase activity was measured 48 hours after transfection. In I-L, GFP expression plasmid was used as control. Data are presented relative to Renilla readings and shown as mean \pm SD of biological triplicates. All results shown are representative of 3 independent experiments. ${ }^{*} P<0.05 ;{ }^{*} P<0.01 ;{ }^{* *} P<0.001$, 2-tailed unpaired Student's $t$ test (D) or 1-way ANOVA with Tukey's multiple comparison test (I-L). 
tional activity by promoting its binding to the target genes. Next, we asked whether XBP1 regulates MYC transcriptional activity. Silencing of XBP1 did not alter the luciferase activities of MYCbinding E box elements in SUM159 cells (Supplemental Figure $5 A)$. Likewise, $X B P 1$ silencing had no effect on the MYC-regulated IRE1 promoter and enhancer activities (Supplemental Figure $5 B)$. Consistently, depletion of XBP1 or IRE1 in SUM159 and MDA-MB-231 cells or overexpression of XBP1s in MCF10A cells did not affect the MYC and MYC-MIZ1 transcriptional programs (Supplemental Figure 5, C and D, and Supplemental Figure 6, A-D). Taken together, these data establish MYC as an important regulator of $\mathrm{XBP} 1 \mathrm{~s}$ by interacting with $\mathrm{XBP} 1 \mathrm{~s}$ and regulating its transcriptional activity. In contrast, XBP1s does not affect MYC transcriptional activity.

MYC hyperactivation is synthetic lethal with XBP1 inhibition. Having established the regulation of the IRE1/XBP1 pathway by oncogenic MYC, we sought to ask whether this interaction between MYC and IRE1/XBP1 could be genetically explored to develop potential new therapies for MYC-driven tumors. To test this hypothesis, we engineered the MCF10A ${ }^{\text {MYC-ER }}$ cells with an XBP1 shRNA under the control of a doxycycline-inducible promoter. Doxycycline treatment efficiently depleted XBP1 in the MCF10A ${ }^{\text {MYC-ER }}$ cells (Supplemental Figure 7A). XBP1 silencing significantly inhibited the clonogenic growth of MYC-hyperactivated cells, while the MYC-low MCF10A ${ }^{\text {MYC-ER }}$ cells showed no response (Figure 5A). The sensitivity of these cells to XBP1 depletion correlated well with the level of MYC in the nucleus (Figure 5, A and B). We found MYC activation dramatically induced the expression of the molecular chaperones (DNAJC10, DNAJC3, DNAJB9, HSPA5), ER protein translocation apparatus (SEC61A1, SEC63), protein modification enzymes (PDIA2, PDIA3), and ERAD components (EDEM1, SEL1L, and SYVN1) that are known to be upregulated by disrupted proteostasis (Supplemental Figure 7C) (1). Silencing of IRE1 or XBP1 blunted the MYC-induced upregulation of these genes (Supplemental Figure 7, B and C). These data demonstrate the importance of the IRE1/XBP1 pathway in mediating the MYCinduced expression of the molecular chaperones, protein modification enzymes, and ERAD.

We also tested the effects of MYC on the PERK and ATF6 branches of the UPR. As shown in Supplemental Figure 8, A and $\mathrm{B}, \mathrm{MYC}$ activation induced PERK downstream eIF2 $\alpha$ phosphorylation and increased CHOP and GADD34 expression. Silencing of PERK substantially suppressed the clonogenic growth of MCF10A ${ }^{\text {MYC-ER }}$ cells, but the effects were independent of MYC hyperactivation (Supplemental Figure 8, C-E). ATF6 levels were not significantly affected by MYC induction (Supplemental Figure 8A). However, ATF6 silencing by 2 shRNA dramatically enhanced the MYC-independent clonogenic growth of MCF10A ${ }^{\text {MYC-ER }}$ cells (Supplemental Figure 8, F-H). Collectively, these data suggest that the 3 arms of UPR play distinct roles in the genetic interaction of UPR with MYC and that only XBP1 inhibition is synthetic lethal with MYC hyperactivation.

The requirement of XBP1 for supporting the survival of MYChyperactivated cells prompted us to evaluate whether pharmacological suppression of XBP1 activation would be beneficial for treating MYC-driven tumors. IRE1 is a dual enzyme with kinase and RNase activities. As its RNase activity is required for $X B P 1$ splicing and activation, we used a fourth-generation salicylaldehyde class inhibitor (8866) that targets the RNase domain of IRE1 (Figure 5C) (33). In $293 \mathrm{~T}$ cells, 8866 inhibited TM-induced XBP1 splicing and the expression of its targets DNAJB9 and SEC61A1 in a dose-dependent manner (Figure 5D and Supplemental Figure $9 \mathrm{~A}$ ). Over $90 \%$ of $X B P 1$ splicing was abolished at $5 \mu \mathrm{M}$ (Figure 5D). Consistently, XBP1 binding to chromatin upon acute ER stress was abrogated by 8866 in SUM159 cells (Figure 5E). Depletion of IRE1 in SUM159 cells dramatically increased the EC50 of 8866 (Supplemental Figure 9B). To confirm a direct interaction between 8866 and IRE1, we performed fluorescence resonance energy transfer (FRET) assays (Figure 5F). We used a soluble truncated enzyme construct containing only the cytosolic kinase and RNase domains of IRE1. A 17-nucleotide-long stem-loop synthetic $X B P 1$ RNA probe with a 6-FAM fluorescent tag on one terminus and a BHQ-1 quencher on the other was used as the substrate. Upon cleavage of the stem loop, the fluorescent tag separated from the quencher, generating a fluorescent signal (Figure 5F). As shown in Figure 5G, 8866 prevented the recombinant IRE1 protein from cleaving the synthetic XBP1 RNA probe, but had no effect on RNase A. RNase L is the sole paralogue of IRE1 in mammals (34, 35), and 8866 did not inhibit its RNase activity either (Supplemental Figure 9C). We also tested the effect of 8866 on the RNase activity of yeast IRE1 (scIRE1). As shown in Supplemental Figure 9D, 8866 did not inhibit yeast IRE1 activities. These data suggest that 8866 is highly selective for mammalian IRE1. To understand whether 8866 inhibits XBP1 splicing by nonselectively targeting IRE1 kinase activity or interfering with general ER stress response, we examined the effects of 8866 on IRE1 phosphorylation and UPR sensor activation. Phosphorylation of IRE1 was not affected by 8866 in 293 T cells treated with TM (Figure 5H). Furthermore, 8866 treatment of $293 \mathrm{~T}$ cells did not affect PERK phosphorylation or ATF6 cleavage (Figure $5 \mathrm{H}$ ), excluding the possibility that the inhibitor interferes with general UPR activation. Collectively, these data confirm that 8866 directly inhibits the RNase activity of mammalian IRE1.

To test whether pharmacological inhibition recapitulates the genetic ablation of $X B P 1$, we treated MCF1OA ${ }^{\mathrm{MYC}-\mathrm{ER}}$ cells with different doses of 4-OHT to induce MYC translocation and examined the effect of 8866 on clonogenicity. Similarly to what occurs in $X B P 1$ depletion (Figure 5A), 8866 selectively restrained the clonogenic growth of MYC-hyperactivated cells (Figure 5I), and the sensitivity of the cells to 8866 correlated with the level of MYC in the nucleus. In addition to XBP1 splicing, the IRE1 RNase also cleaves ER-localized mRNAs through the XBP1-independent regulated IRE1-dependent decay (RIDD) pathway under excessive ER stress $(1,36)$. To test whether 8866 inhibits RIDD, we treated 293T cells with TM to induce IRE1 hyperactivation and tested the response of RIDD targets to various doses of 8866. As shown in Supplemental Figure 9E, TM treatment of 293T cells substantially reduced the level of RIDD targets CD59, DGAT2, and PMP22. Treatment with 8866 completely reversed the degradation of these RIDD targets, suggesting that 8866 also inhibits RIDD. Next, we asked whether MYC activation is sufficient to induce RIDD activation and whether the treatment of MYC-dependent breast cancer cells with 8866 inhibits the degradation of RIDD targets. As shown in Supplemental Figure 2D, dose-dependent activation of MYC did not induce 
A $4-\mathrm{OHT}(\mathrm{nM})$

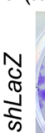

论

0

5

10

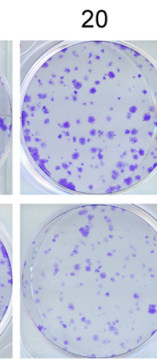

50
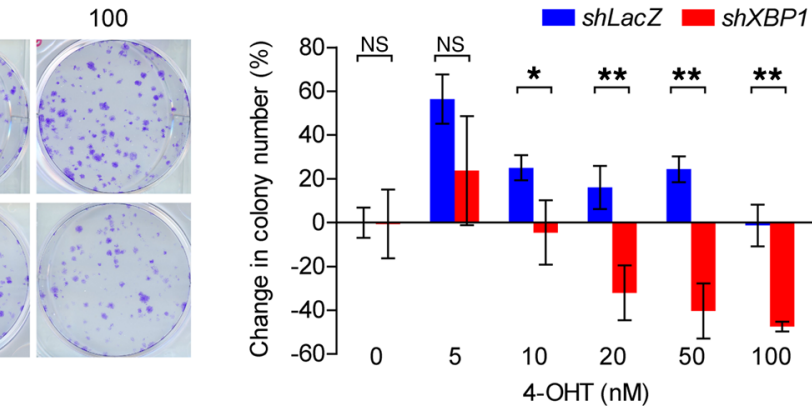

B

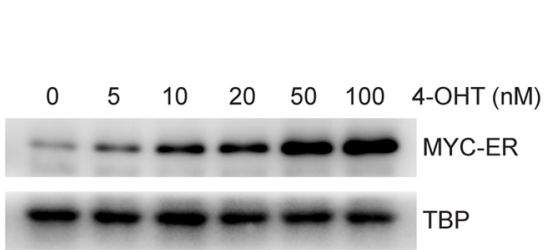

C

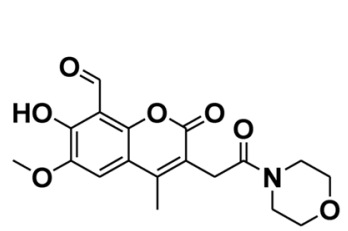

8866
D

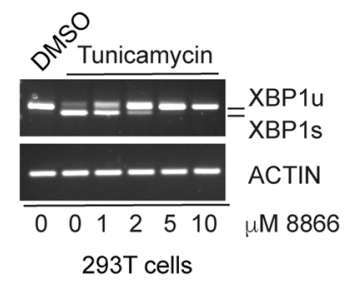

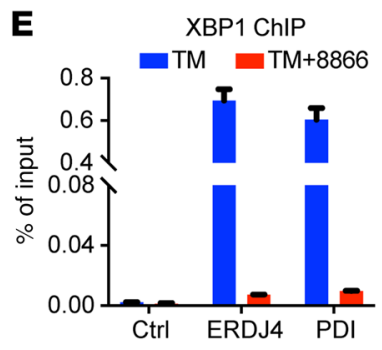
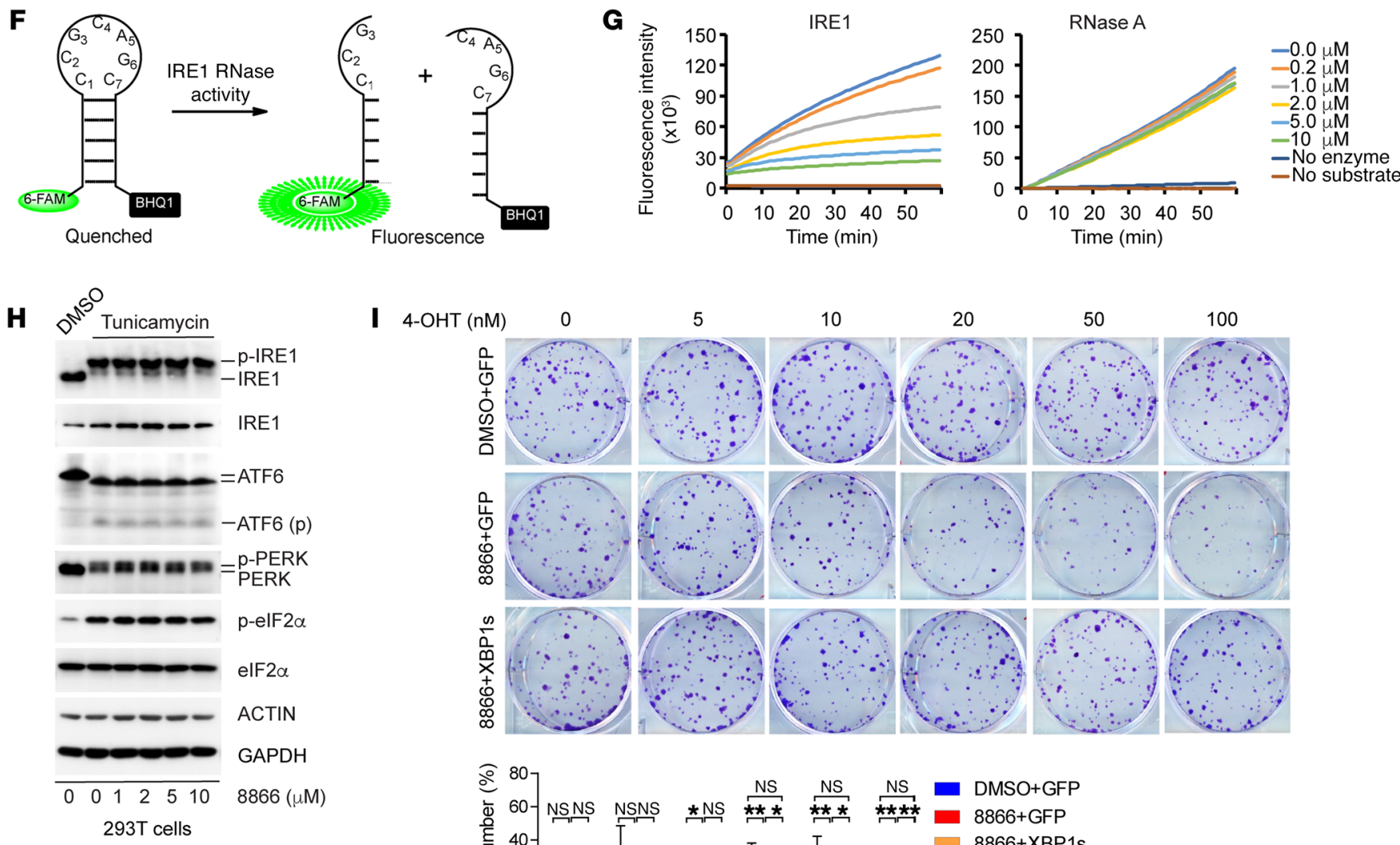

10

20

50 100
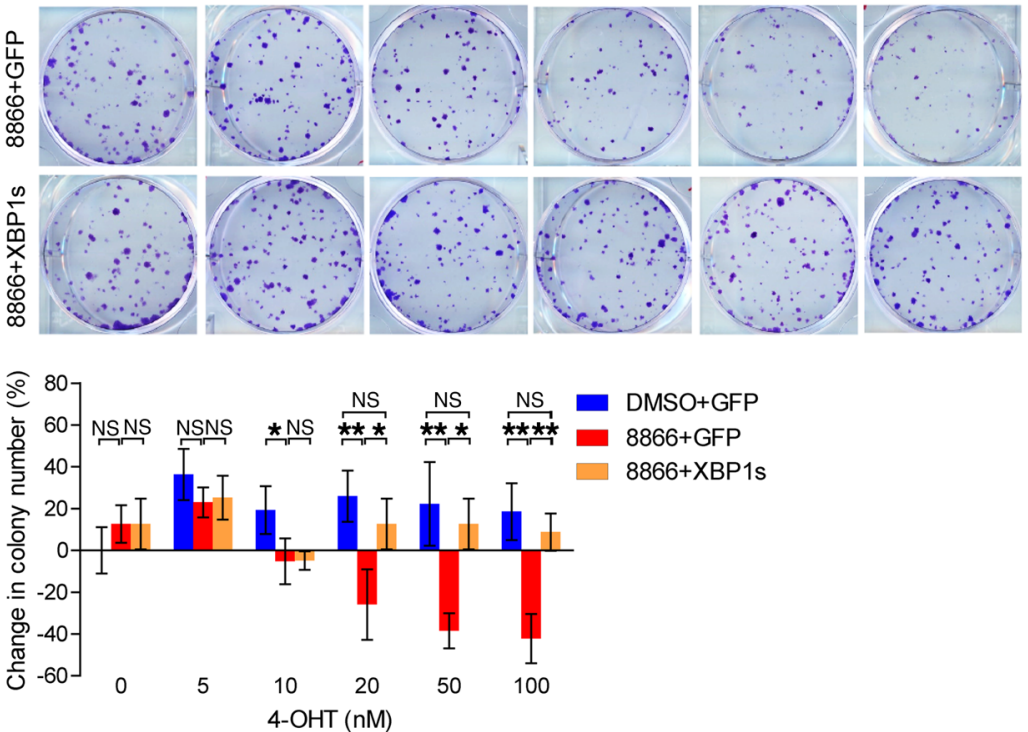
Figure 5. MYC hyperactivation is synthetic lethal with XBP1 inhibition. (A) Clonogenic growth of MCF10A ${ }^{\text {MYC-ER }}$ cells transduced with shRNAs against $X B P 1$ or $L a c Z$ and treated with different doses of 4-OHT. Ethanol was used as vehicle for 4-OHT. Changes in colony number were compared with vehicle-treated cells expressing shLacZ. (B) Immunoblot of MYC-ER in nuclear extracts of MCF10A ${ }^{\text {MYC-ER }}$ cells treated with 4-OHT for 24 hours. (C) Chemical structure of 8866. (D) XBP1-splicing assay in 293T cells that were treated with different doses of 8866 in the presence of DMSO or $5 \mu \mathrm{g} / \mathrm{ml}$ TM for 6 hours. (E) SUM159 cells were treated with DMSO or $5 \mu$ M 8866 in the presence of $5 \mu \mathrm{g} / \mathrm{ml}$ TM for 6 hours. ChIP assays were performed using anti-XBP1s antibody. Data are presented relative to input and shown as mean \pm SD of technical triplicates. (F) Schematic diagram of fluorescencebased RNA cleavage assay. (C) Cytosolic portions of IRE1 protein or RNase A were incubated with hairpin XBP1 RNA substrate in the presence of various doses of 8866 . Cleavage reactions were monitored by fluorescence intensity. (H) Immunoblot of IRE1 phosphorylation (phos-tag SDS-PACE), ATF6 cleavage (ATF6p), PERK, and elF2 $\alpha$ phosphorylation in 293T cells treated with different doses of 8866 for 6 hours in the presence of DMSO or $5 \mu \mathrm{g} / \mathrm{ml} \mathrm{TM}$. Images shown are representative of 3 independent experiments. (I) Clonogenic growth of MCF10A ${ }^{M Y C-E R}$ cells transduced with GFP or XBP1s and treated with DMSO or $5 \mu \mathrm{M} 8866$ in the presence of different doses of 4-OHT. Changes in colony number were compared with

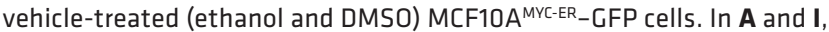
data are presented as mean \pm SD of biological triplicates. ${ }^{*} P<0.05$; ${ }^{*} P<0.01$, 2-tailed unpaired Student's $t$ test (A) or 1-way ANOVA with Tukey's multiple comparison test (I).

the degradation of RIDD targets CD59, DGAT2, and PMP22. Furthermore, treatment of MYC-dependent SUM159 cells with 8866 had no effects on these RIDD targets (Supplemental Figure 9F). These data suggest, that although 8866 inhibits RIDD, RIDD is not activated in MYC-driven breast cancer cells.

To directly test whether the impact of 8866 on the clonogenic growth of MYC-hyperactivated cells is due to the modulation of off-targets, we ectopically expressed XBP1s in 8866-treated MCF10A ${ }^{\text {MYC-ER }}$ cells and performed clonogenic assays. Enforced expression of XBP1s completely rescued the 8866-mediated growth inhibition of the MYC-hyperactivated cells (Figure 5I), indicating that the effects of IRE1 RNase inhibitor 8866 on MYChyperactivated cells are mediated by XBP1 splicing.

Taken together, these data indicate that MYC hyperactivation is synthetic lethal with IRE1 RNase inhibition and that both genetic and pharmacological inhibition of XBP1 activation selectively suppress the growth of MYC-hyperactivated cells.

8866 Suppresses growth of PDX and GEM tumors with high MYC expression and sensitizes the response to chemotherapy. To examine the efficacy of IRE1 inhibition of XBP1 splicing for preventing MYC-driven tumor progression in vivo, we utilized PDX models, which recapitulate the patient tumor heterogeneity and histopathology and have been used to predict the clinical response of patients to drug treatment (37). We first tested different doses of 8866 for maintaining in vivo suppression of the IRE1/XBP1 pathway. $X b p 1$ splicing and its target gene expression were continuously inhibited in mice administered $300 \mathrm{mg} / \mathrm{kg}$ of 8866 via daily oral gavage (Supplemental Figure 10, A and B), a dose that was then selected for evaluating its efficacy in 4 preclinical in vivo tumor models with differing levels of MYC protein expression (MC1, 4913, 2147, and 4195) (Figure 6, A and B). MC1 PDX tumors, which expressed the highest level of MYC, were orthotopically transplanted into the cleared mammary fat pads of SCID/beige mice and allowed to grow to an average size of $300 \mathrm{~mm}^{3}$. The animals with PDX tumors were then randomly grouped and treated with 8866 or a vehicle control daily via oral gavage (Figure 6C). Treatment with 8866 potently inhibited the growth of MC1 PDX tumors and extended the survival of the tumor-bearing mice (Figure 6, D and E). We confirmed that XBP1 splicing and the expression of XBP1 targets were suppressed by 8866 treatment via analysis of RNA extracted from the tumor tissues (Figure 6, F and G). Furthermore, tumors from 8866-treated animals showed significant reduction of BrdU incorporation after 4 days of treatment (Supplemental Figure 12, A and B). We also observed a reduced number of $\mathrm{CD} 31^{+}$microvessels and an increase in cleaved caspase- 3 staining after 20 days of treatment (Figure 6, H-J). These data indicate that 8866 impaired proliferation and angiogenesis and promoted apoptosis. Treatment of the tumor-bearing mice with $8866 \mathrm{did}$ not cause significant body weight change or obvious signs of toxicity in major organs (Supplemental Figure 10, C and D).

To assess whether the treatment response to 8866 is correlated with MYC levels, we performed similar experiments with the 3 other PDX models expressing decreasing levels of MYC, i.e., models 4913, 2147, and 4195. Treatment with 8866 suppressed the growth of established tumors in model 4913 and extended the survival of tumor-bearing mice (Figure 6K). However, the effectiveness was reduced in models with lower MYC expression. Although the inhibitor treatment slowed the tumor progression in model 2147, it was less effective (Figure 6L). Furthermore, treatment was ineffective in model 4195, which expressed the lowest levels of MYC, comparable to those of normal human breast tissues (Figure 6, A and M). Notably, treatment of all 3 PDX models with 8866 did not result in significant body weight change or cause obvious signs of toxicity in major organs (Supplemental Figure 10, E-I).

Next, we asked whether increasing the MYC levels in the 4195 PDX model could sensitize the tumors to 8866 treatment. While it is technically difficult to genetically manipulate PDX tumors, we used a pharmacological approach to transiently induce MYC protein levels in 4195 PDX tumors. Phosphorylation of MYC on Thr-58 by GSK3 $\beta$ facilitates the binding of FBW7 to MYC and induces FBW7-mediated MYC ubiquitination and degradation $(38,39)$. Pharmacological inhibition of GSK3 $\beta$ with a small molecule CHIR-99021 has been shown to stabilize MYC protein (40). We treated 4195 tumors with vehicle, CHIR-99021, 8866, and 8866 plus CHIR-99021 and assessed the tumor response. As shown in Supplemental Figure 11A, treatment of 4195 tumors with CHIR-99021 modestly induced MYC protein levels. The CHIR-99021-treated tumors were partially responsive to 8866 treatments (Supplemental Figure 11B). These data indicate that transient increased MYC levels partially sensitized the tumors to 8866 treatment. Collectively, these data demonstrate the in vivo efficacy of 8866 for suppressing tumor growth and indicate that the IRE1/XBP1 pathway is important for the tumorigenicity of MYC-overexpressing tumors.

With these encouraging results, we next sought to determine whether 8866 enhances the effectiveness of standard chemotherapy treatment. Currently, chemotherapy is the only systemic therapy used clinically to treat TNBC. However, patients with TNBC either have limited response rates or relapse within 1 to 3 years (41). For these experiments, we again utilized SCID/beige 
A

B
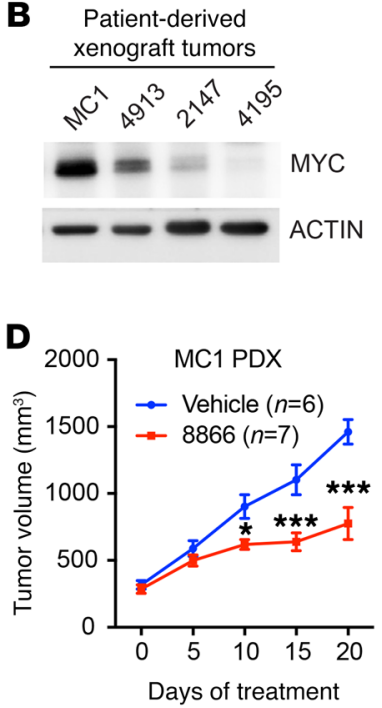

E

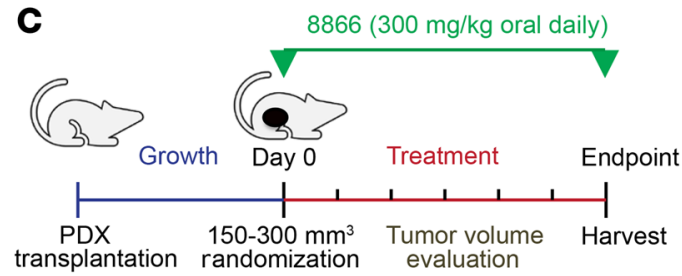

F

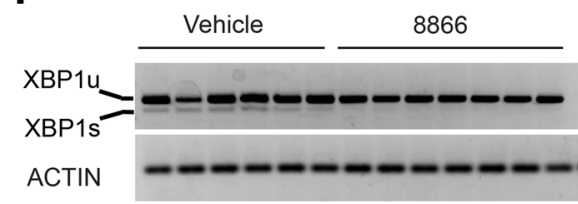

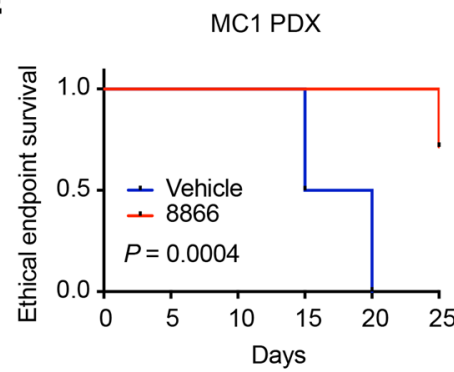

G
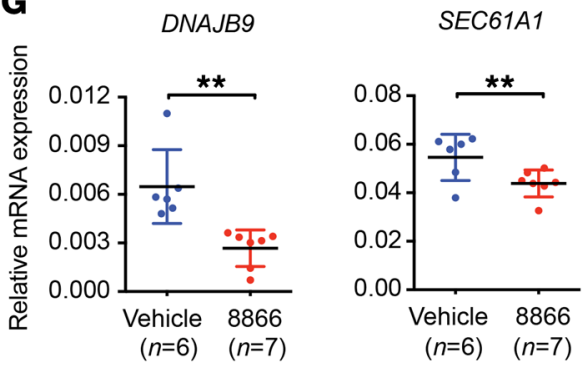

SEC61A1

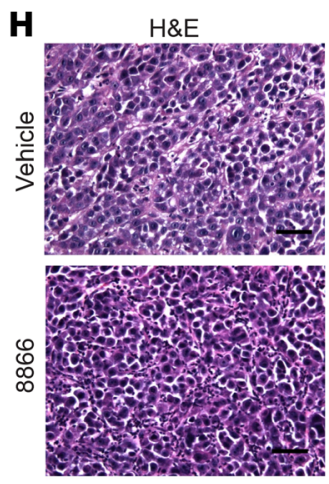

J Cleaved Caspase-3

Cleaved Casp-3

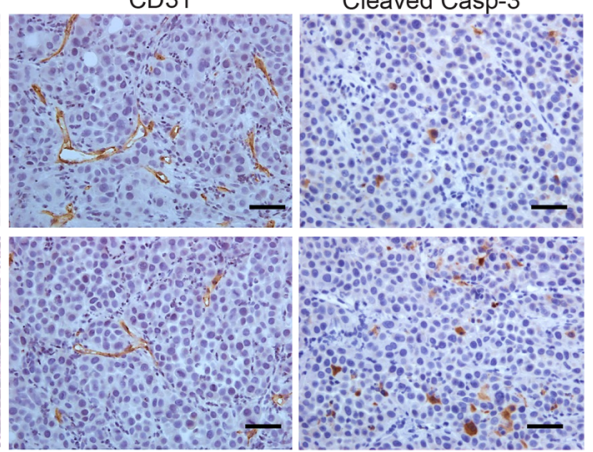

I

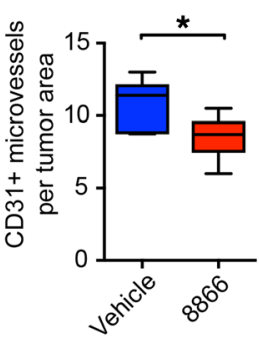

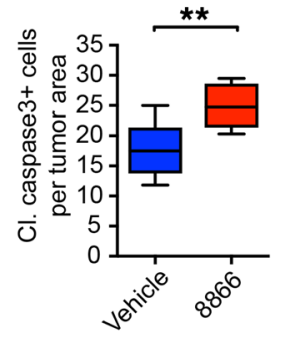
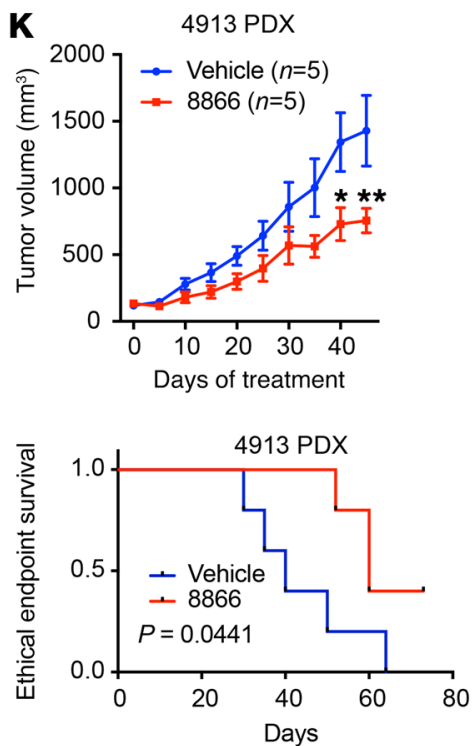
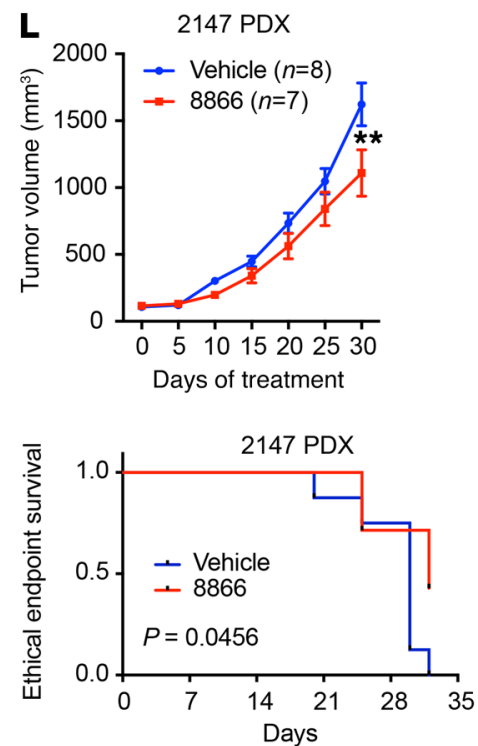
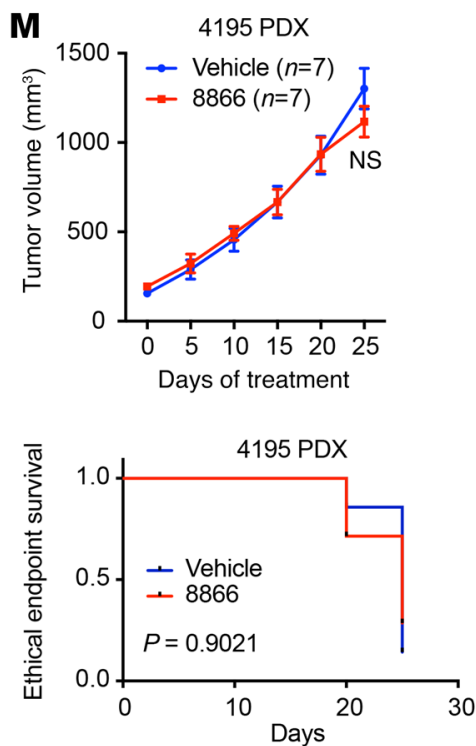
Figure 6. IRE1 RNase inhibitor 8866 suppresses growth of patientderived tumors with high MYC expression. (A) Immunostaining of MYC in human normal mammary gland or PDX tumors. Normal mammary gland without primary antibody incubation was used as negative control. Scale bars: $50 \mu \mathrm{m}$. (B) Immunoblot of MYC in PDX models. (C) Schematic of treatment strategy with 8866. (D) Tumor volume quantification of established MC1 PDX tumors in SCID/beige mice treated with vehicle or 8866. Data are presented as mean \pm SEM. (E) Kaplan-Meier survival curve of MC1 PDX tumor-bearing mice from treatment start time in vehicle ( $n=$ 6) and $8866(n=7)$ treatment groups. (F) RT-PCR analysis of XBP1 splicing in vehicle-treated $(n=6)$ and 8866-treated $(n=7)$ MC1 PDX tumor samples harvested at the end of the experiment. (C) qRT-PCR analysis of XBP1 target gene expression in vehicle-treated and 8866-treated MC1 PDX tumor samples harvested at the end of the experiment. Data are presented as mean \pm SD of biological replicates, and actin was used as internal control. (H) H\&E, cleaved caspase-3, or CD31 immunostaining of MC1 PDX tumors harvested at the end of the experiment. Scale bars: 50 $\mu \mathrm{m}$. (I and J) Quantification of CD31-positive cells (I) or cleaved caspase-3-positive cells (J) on tumor sections from vehicle-treated $(n=6)$ or 8866 -treated ( $n=7$ ) MC1 PDX mice. (K-M) Tumor volume quantification (upper panel) and Kaplan-Meier survival curve (lower panel) of 4913 (K), 2147 (L), and 4195 (M) PDX tumor-bearing mice treated with vehicle or 8866. Data are presented as mean \pm SEM. The log-rank test was used to test for the significant differences of survival between the groups $(\mathbf{E}$, K-M). ${ }^{*} P<0.05$; ${ }^{* *} P<0.01$; ${ }^{* *} P<0.001$, 2-way ANOVA with Bonferroni's post test (D, K-M) or 2-tailed unpaired Student's $t$ test $(\mathbf{G}, \mathbf{I}, \mathbf{J})$.

mice with orthotopically transplanted MC1 PDX tumors grown to 300 to $400 \mathrm{~mm}^{3}$. Mice were then divided into 1 of 4 groups to receive treatment with vehicle, with 8866 alone, with docetaxel alone, or with a combination of the 2 drugs (Figure 7A). Tumor growth was reduced in mice treated with docetaxel, but no tumor regression was observed (Figure 7B). Similarly, tumors in mice treated with 8866 progressed more slowly than those treated with control (Figure 7B). However, treatment with a combination of docetaxel and 8866 resulted in rapid tumor regression within 10 days, and no palpable tumors were observed after 30 days (Figure 7B). The combination treatment was stopped after 42 days, and no tumor recurrence was observed afterwards. Immunohistochemical analyses showed a remarkable decrease of BrdU incorporation after 4 days of treatment (Supplemental Figure 12, A and B) and significant increases in apoptosis after 20 days of treatment in the combination treatment group (Figure 7, C and D, and Supplemental Figure 12C). These data indicate that 8866 potently sensitizes the tumors to the apoptotic and proliferation inhibitory effects of chemotherapy. Moreover, treatment with 8866 alone or in combination with docetaxel substantially prolonged the survival of the tumor-bearing mice (Figure 7E). No signs of tissue damage were detected upon examination of the liver, pancreas, kidney, lung, heart, and small intestine (Supplemental Figure 12D). To further assess safety, we tested apoptosis in the liver and pancreas of the tumor-bearing mice. Neither 8866 alone nor combination therapy with docetaxel increased cell death in liver and pancreas (Supplemental Figure 13, A-D). Food intake and glucose levels were unaltered upon treatment (Supplemental Figure 14, A and B). Consistent with previous studies, inhibition of Xbp1 splicing by 8866 resulted in increased Ire1 protein in liver and pancreas (Supplemental Figure 13, A and B) (42). The treatments did not activate other UPR sensors (Supplemental Figure 13, A and B). It is noteworthy that combination therapy with 8866 and docetaxel led to a transient reduction of body weight during rapid tumor regression, but that the body weight was restored after tumors were eradicated (Supplemental Figure 14C). This reduction in body weight, therefore, was likely due to the combined effects of significant tumor weight reduction and potential transient toxicity caused by rapid tumor lysis (43).

Since PDX models lack an intact immune system, we tested the therapeutic efficacy of 8866 , alone or in combination with chemotherapy, in 2 syngeneic p53-null GEM models expressing distinct levels of Myc (Figure 7F). p53 is mutated in approximately $85 \%$ of basal-like breast cancers (44). Despite the common loss of $p 53$, the $p 53^{-/-}$GEM models exhibited remarkable intertumoral heterogeneity in genomic copy number, gene-expression profiles, and histopathology, reminiscent of human breast cancers (44-46). As shown in Figure 7G, while 8866 alone slowed down the progression of Myc-overexpressing 2153L GEM tumors, combination therapy with 8866 and docetaxel synergistically blocked tumor growth. The Myc-low-expressing T11 GEM tumors showed no response to the treatments (Figure $7 \mathrm{H}$ ). Immune surveillance plays essential roles in tumor progression (47). To test whether treatment with 8866 , alone or in combination with docetaxel, affects immune surveillance, we analyzed the $2153 \mathrm{~L}$ and T11 GEM tumors for the infiltration of T cells, B cells, myeloid-derived suppressor cells (MDSCs), macrophages, and DCs. Interestingly, while 8866 alone minimally affected immune surveillance in both 2153L and T11 GEM models, we observed significantly increased infiltration of $\mathrm{CD}^{+}$and $\mathrm{CD} 8^{+} \mathrm{T}$ cells and marked decrease of MDSCs selectively in Myc-overexpressing 2153L GEM tumors (Supplemental Figure $15, \mathrm{~A}$ and $\mathrm{B})$. These data indicate that combination therapy with 8866 and docetaxel is effective in suppressing Myc-overexpressing GEM tumor progression and enhancing immune surveillance in tumors. Similarly to what occurred in the MC1 PDX model, combination therapy led to reduced body weight of the mice bearing 2153L tumors after 9 days of treatment, which was likely due to the substantially decreased tumor weight (Supplemental Figure 15C). The same treatments did not cause body weight alteration of the nonresponsive T11 GEM model (Supplemental Figure 15D).

Having established the therapeutic efficacy of 8866 alone or in combination with docetaxel in primary tumors, we next asked whether the treatments are effective in blocking breast cancer metastasis. We inoculated the MDA-MB-231-LM2 cells into the athymic nude mice via tail-vein injection and randomly grouped the mice for treatments with vehicle, with 8866 alone, with docetaxel alone, or with a combination of the 2 drugs. While 8866 alone had little effect on lung metastasis, combination therapy markedly blocked the metastasis of MDA-MB-231-LM2 cells to the lung (Supplemental Figure 16, A and B). Collectively, these findings using multiple preclinical breast cancer models reveal that pharmacological inhibition of the IRE1/XBP1 pathway with an IRE1 RNase inhibitor prevents MYC-hyperactivated tumor growth and sensitizes the tumors to standard-of-care chemotherapy.

\section{Discussion}

MYC deregulation is one of the most common drivers of human cancers (30). The direct targeting of MYC is very challenging and has been unsuccessful to date. MYC hyperactivation results in enormous transcriptional and translational perturbations. The 
A

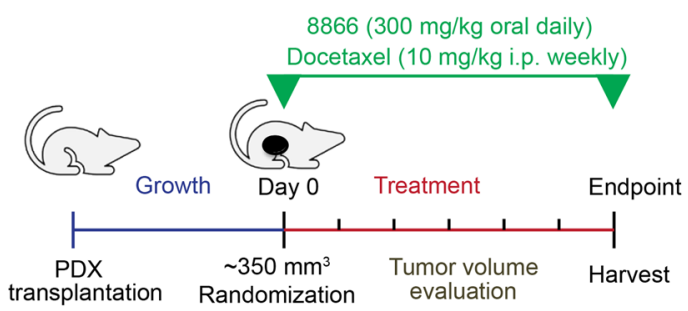

B

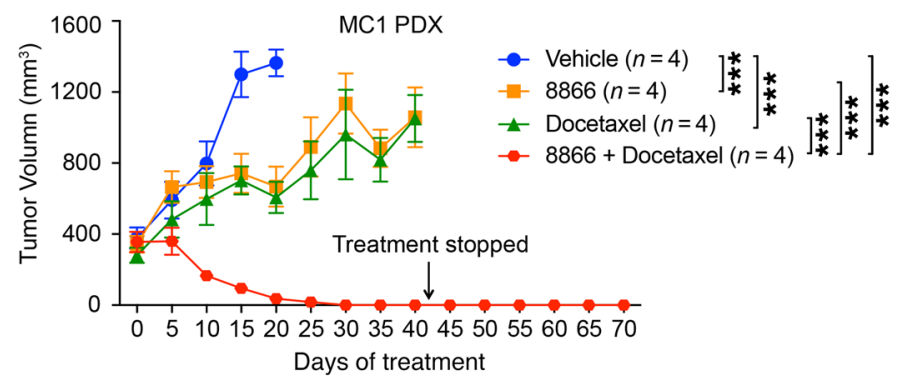

C

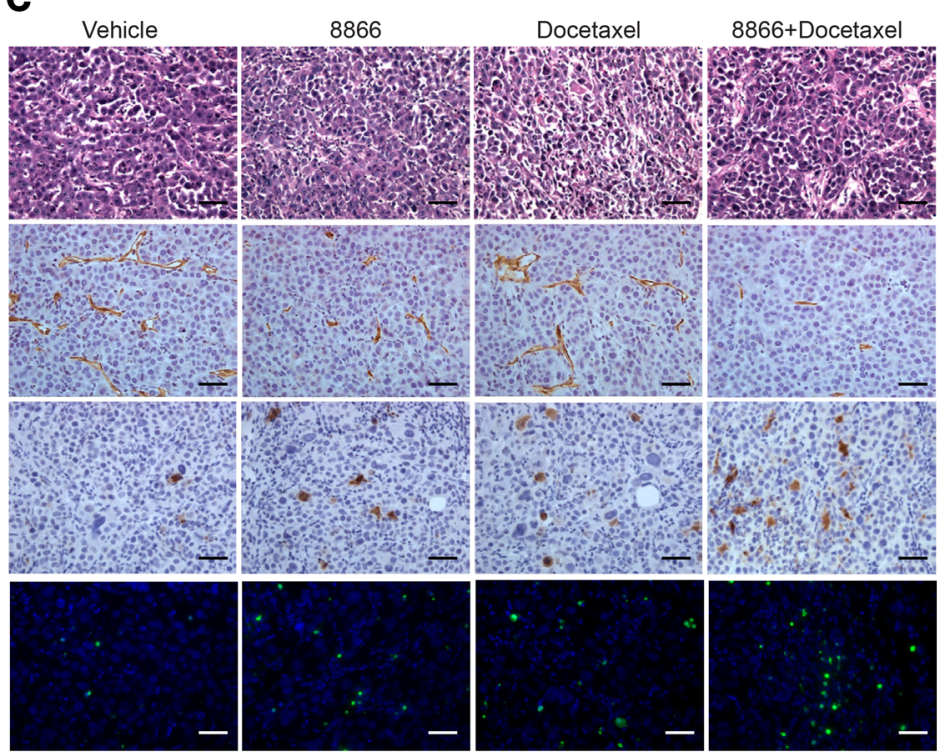

D
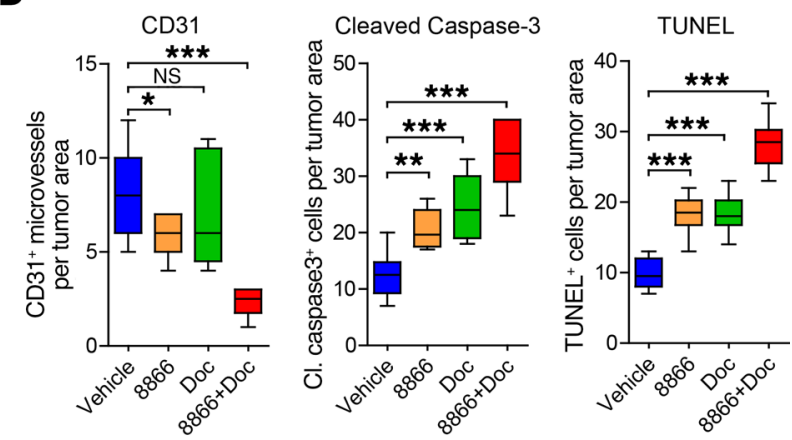

E

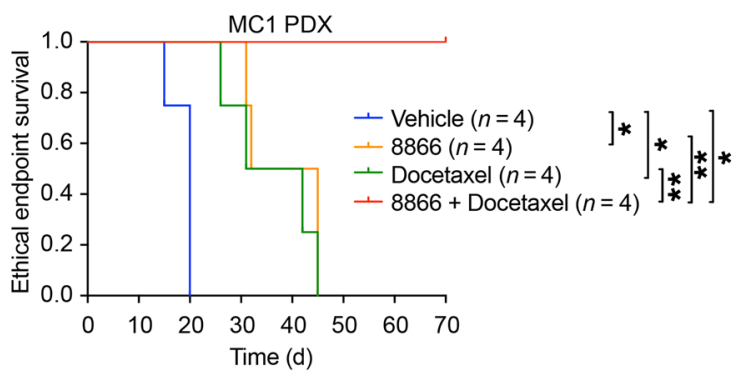

$\mathbf{F}$

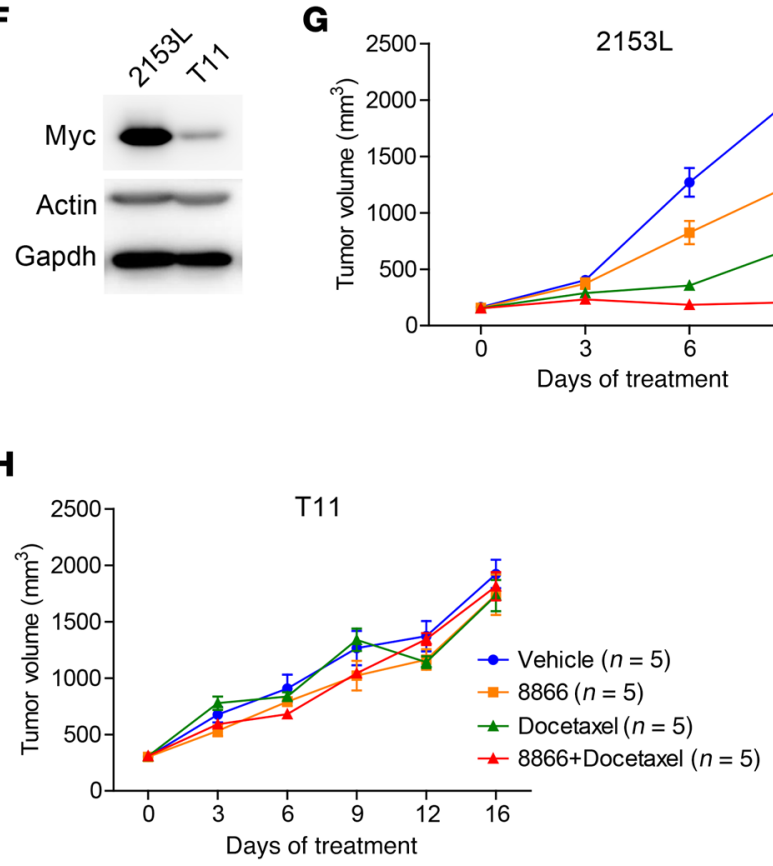

I

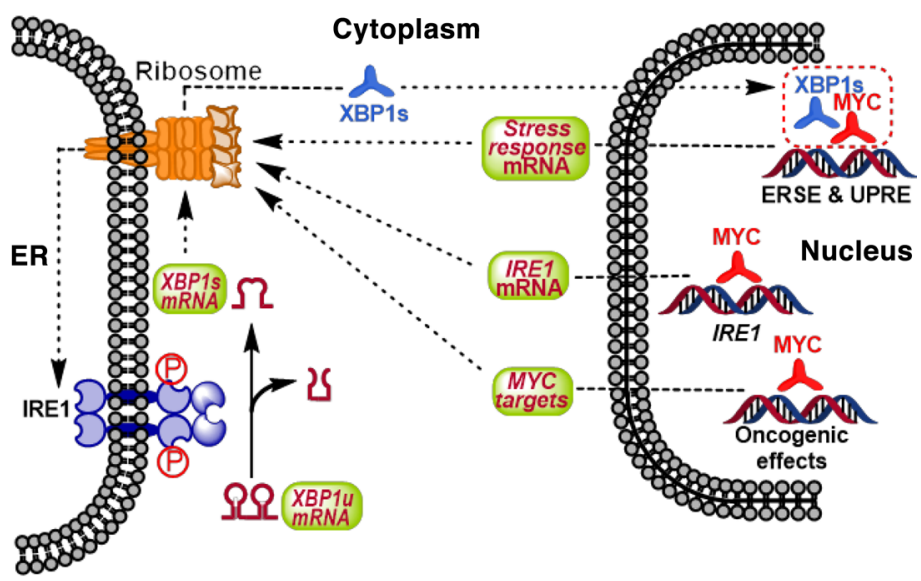


Figure 7. 8866 enhances MYC-overexpressing PDX and GEM tumor response to docetaxel chemotherapy. (A) Schematic of treatment strategy with 8866 with or without docetaxel. (B) Tumor volume quantification of established MC1 PDX tumors in SCID/beige mice treated with vehicle, 8866 , docetaxel, or 8866 plus docetaxel $(n=4)$. Combination treatment of tumor-bearing mice with 8866 plus docetaxel was stopped at day 42 . Data shown are representative of 3 independent experiments and presented as mean \pm SEM. (C) H\&E, immunostaining of CD31 or cleaved caspase-3 (Casp-3), and TUNEL staining of MC1 PDX tumors in different treatment groups harvested 20 days after treatment. Representative images are shown. Scale bars: $50 \mu \mathrm{m}$. (D) Quantification of CD31-positive microvessels, cleaved caspase-3-positive cells, or TUNEL-positive cells on tumor sections from different treatment groups. Doc, docetaxel. 6-12 tumor areas from each group were counted. (E) Kaplan-Meier survival curve of MC1 PDX tumor-bearing mice from treatment start time in vehicle, 8866, docetaxel, or 8866 plus docetaxel treatment groups. (F) Immunoblot of Myc in tissue lysates of 2 p53-null GEM models. Actin and GAPDH were used as loading controls. ( $\mathbf{G}$ and $\mathbf{H}$ ) Tumor volume quantification of established $2153 \mathrm{~L}$ $(\mathbf{G}, n=6)$ and $\mathrm{T} 11(\mathbf{H}, n=5)$ tumors in BALB/c mice treated with vehicle, 8866 , docetaxel, or 8866 plus docetaxel. (I) Model for the role of the IRE1/ XBP1 pathway in MYC-driven breast cancer. Oncogenic MYC activates IRE1 transcription and forms a transcriptional complex with XBP1 to facilitate the resolution of MYC-induced proteotoxic stress and the restoration of ER homeostasis. Data are presented as mean \pm SEM. ${ }^{*} P<0.05$; ${ }^{*} P<0.01$; ${ }^{* * *} P<0.001,2$-way ANOVA with Bonferroni's post test (B and $\mathbf{H}$ ), 1-way ANOVA with Tukey's multiple comparison test (D) or log-rank test (E).

MYC-driven escalation of protein synthesis alters the proportions of protein subunits in protein complexes (48), induces the accumulation of unfolded and misfolded proteins, and confers extensive proteotoxic stress (49). Understanding how MYC-hyperactivated cancer cells tolerate this proteotoxic stress and maintain proteostasis is imperative for identifying the Achilles' heel that could be exploited for therapeutics. In this study, we show that the IRE1/ XBP1 pathway is a therapeutic vulnerability accompanied by MYC hyperactivation in breast cancer. MYC is substantially involved in the UPR regulation via multiple previously unknown mechanisms for coping with proteotoxic stresses.

It is known that MYC-induced protein synthesis and ribosome biogenesis can indirectly activate the PERK branch of the UPR by increasing the ER protein load in lymphomas (49). Our study and a companion study by Simon and colleagues (50) demonstrate an unprecedented direct transcriptional regulation of another ER stress sensor, IRE1, by MYC in breast cancer and other cancers, including Burkitt's lymphoma and neuroblastoma. MYC directly binds to and regulates the IRE1 promoter and enhancer regions. Silencing of MYC in MYC-dependent cancer cells decreased IRE1 expression and suppressed $X B P 1$ splicing. Unexpectedly, we found that MYC also directly interacts with XBP1 in the nucleus and enhances its transcriptional activity, thereby increasing the expression of ER molecular chaperones and protein modification and folding enzymes. In contrast, XBP1 does not affect the MYC transcriptional program. Collectively, although MYC deregulation results in extensive proteotoxic stress and the perturbation of ER homeostasis, it directly facilitates the resolution of this stress by hijacking the IRE1/ XBP1 arm of the UPR at multiple levels (Figure 7I). In this way, MYC aids in the correct folding and modification of newly synthesized proteins as well as in the elimination of toxic misfolded proteins by inducing the IRE1/XBP1-dependent expression of molecular chaperones, protein modification enzymes, and ERAD components.
Intriguingly, our genetic approaches demonstrate the importance of these counteracting activities of MYC in cancer cells. MYC hyperactivation is synthetic lethal with $X B P 1$ suppression. We, therefore, exploited this therapeutic window with a pharmacological approach to targeting XBP1. Chemical inhibition of IRE1 is the most attractive therapeutic strategy for inhibiting XBP1 signaling, since its dual enzymatic functions allow interference at multiple mechanistic levels of activation. Targeting the IRE1 RNase domain offers a unique therapeutic strategy. Several IRE1 RNase inhibitors have been patented and published, including $4 \mu 8 \mathrm{c}$, MKC3946, STF083010, and B-IO9 (5, 18, 19, 33, 51). Although MKC3946 and STF083010 have shown in vivo efficacy in multiple myeloma cell line xenograft models (5, 19), treatment of the tumors with inhibitor alone was unable to regress solid tumors. Furthermore, to date, the in vivo validation of UPR inhibitors in patient-relevant preclinical models is still lacking. It is unclear what population of patients would respond to UPR inhibitors. We now show that the IRE1 RNase inhibitor 8866 directly inhibits IRE1 RNase activity. Although 8866 inhibits both RIDD and XBP1 splicing, MYC induction does not activate RIDD in breast cancer cells. The effects of 8866 on MYC-hyperactivated cells are mediated by XBP1 splicing, as restoration of XBP1s completely rescued the phenotype. More importantly, 8866 showed in vivo efficacy in preclinical models. Using a cohort of PDX models, we showed that the progression of MYC-overexpressing patient-derived tumor MC1 was delayed with 8866 treatment with a comparable efficacy to that of the standard-of-care chemotherapy docetaxel. Remarkably, treatment with a combination of 8866 and docetaxel rapidly eradicated the primary tumors with high MYC expression, which showed a marked reduction of proliferation and induction of apoptosis, thereby substantially prolonging the disease-free survival of tumor-bearing mice. We did not observe any tumor recurrence at least 30 days after ending the combination treatment. Importantly, treatment of these mice with 8866 continuously for 42 days did not cause obvious signs of major organ toxicity or significant body weight change. Similar safety profiles were also observed in rats and monkeys (data not shown). While 8866 alone did not affect the body weight of MC1 tumor-bearing mice, combination therapy with 8866 and docetaxel led to transient body weight reduction. However, body weight quickly restored after tumor eradication and we did not observe tissue damage in the major organs. Furthermore, treatment of the nonresponsive T11 GEM model with combination therapy did not cause any body weight alteration. These data indicate that the transient body weight loss in the treatment-sensitive models is likely due to the substantial tumor mass reduction and transient toxicity associated with rapid tumor lysis (43). Remarkably, the combination therapy was also very effective in blocking Mycoverexpressing GEM tumor growth and enhancing immune response in the tumors. Taking these data together, we identify MYC-overexpressing breast tumors as potential responders to 8866 treatment, alone or in combination with chemotherapy. Our study delineates the genetic interactions between MYC and IRE1/XBP1 and reveals the critical role of IRE1/XBP1 in supporting the growth of MYC-driven breast cancer. A companion study by Simon and colleagues independently showed a similar require- 
ment of IRE1/XBP1 signaling for MYC deregulation in Burkitt's lymphoma and neuroblastoma (50). These 2 studies suggest there may be a potentially significant clinical impact of targeting IRE1/XBP1, as we provide a mechanism-based pharmacological approach for the treatment of MYC-driven human tumors.

\section{Methods}

Cell culture and treatment. MDA-MB-231, 293T, and BT-549 cells were obtained from ATCC and maintained in DMEM or RPMI supplemented with 10\% FBS (catalog A3160602; Gibco, Thermo Fisher Scientific) and penicillin/streptomycin. The MCF10A cells were from ATCC and cultured in DMEM/F12 medium, $20 \mathrm{ng} / \mathrm{ml}$ epidermal growth factor (EGF) (catalog PHG0311L; Gibco, Thermo Fisher Scientific), 10 $\mu \mathrm{g} / \mathrm{ml}$ insulin (catalog 19278, MilliporeSigma), 2\% horse serum, 500 $\mathrm{ng} / \mathrm{ml}$ hydrocortisone (catalog H0888, MilliporeSigma), $100 \mathrm{ng} / \mathrm{ml}$ cholera toxin (catalog C8052, MilliporeSigma), and penicillin/streptomycin. SUM159 cells were obtained from Asterand and maintained in F12 medium supplemented with 5\% FBS, $10 \mu \mathrm{g} / \mathrm{ml}$ insulin, $500 \mathrm{ng} / \mathrm{ml}$ hydrocortisone, and penicillin/streptomycin.

4-OHT (catalog H7904-5MG, MilliporeSigma) was used to treat

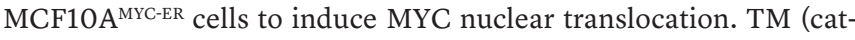
alog T7765, MilliporeSigma) was used to treat cells or mice for ER stress induction. IRE1 RNase inhibitor 8866 was dissolved in DMSO when used in vitro.

For the clonogenic assay of MCF10A ${ }^{\mathrm{MYC}-\mathrm{ER}}$ cells, 500 cells were plated in a 6-well plate and treated with either vehicle (ethanol) or 4-OHT for 8 to 10 days. Colonies were fixed in methanol, stained with crystal violet (0.5\% crystal violet, $20 \%$ methanol), and counted.

Plasmids, virus production, and infection. pLKO.1-shMYC-1 (TRCN0000039640) and pLKO.1-shMYC-2 (TRCNO000039639) were obtained from MilliporeSigma. shRNAs targeting scramble RNA (pLKO.1-shScr, Addgene plasmid 17920) served as a control. Inducible XBP1/IRE1/PERK/ATF6 shRNA or control shRNA (shLacZ) was cloned in $\mathrm{PLKO}$-Tet-On lentiviral vector. The sequences of shRNA are listed in Supplemental Table 1. Retroviral vector pQCXIN-MycER was used to generate the MCF1OA ${ }^{\mathrm{MYC}-\mathrm{ER}}$ cell line.

To produce lentiviruses, 293T cells were cotransfected with psPAX2 and pMD2.G using Lipofectamine 3000 (catalog L3000015, Thermo Fisher Scientific). To produce retrovirus, pQCXIN-MycER and pCL-Ampho plasmids were cotransfected into 293T cells. Lentior retroviruses were collected 48 and 72 hours after transfection and used for infecting cells in the presence of $8 \mu \mathrm{g} / \mathrm{ml}$ polybrene (catalog TR-1003-G, MilliporeSigma) prior to puromycin ( $2 \mu \mathrm{g} / \mathrm{ml}$ for all cells, catalog P8833, MilliporeSigma) or G418 (200 $\mu \mathrm{g} / \mathrm{ml}$ for MCF10A, catalog 4727878001, MilliporeSigma) selection.

Generation of KO cells. An inducible CRISPR/Cas9 system (52) was used to generate multiclonal IRE1- or XBP1-KO SUM159 cells. Briefly, SUM159 cells were infected with lentiviruses encoding doxycyclineinducible Cas9-EGFP (gift from Qin Yan, Yale University, New Haven, Connecticut, USA). The cells were treated with doxycycline, followed by FACS sorting for $\mathrm{EGFP}^{+}$cells. The resulting SUM159-iCas9 cells were infected with lentiviruses expressing double gRNAs targeting the same exon and treated with doxycycline to induce Cas9 expression and gene KO.

To generate IRE1- or XBP1-KO MDA-MB-231 cells, gRNAs targeting IRE1- or XBP1 were introduced to the pSpCas9 (BB)-2A-Puro (PX459) lentiviral vector (plasmid 62988, Addgene). MDA-MB-231 cells were infected with lentiviruses encoding IRE1 or XBP1 sgRNA and selected with puromycin. Single cells were derived from the cultures and $4 \mathrm{KO}$ colonies were pooled.

RNA extraction and real-time quantitative reverse-transcriptase PCR. Total RNA was extracted using TRIzol (catalog 15596026, Thermo Fisher Scientific). Total RNA (1 $\mu \mathrm{g})$ was converted to cDNA using the High-Capacity cDNA Reverse Transcription Kit (catalog 4368813, Applied Biosystems). mRNA levels were detected using Power SYBR Green PCR Master Mix (Applied Biosystems) on a QuantStudio 6 Flex Real-Time PCR System (Applied Biosystems). The sequences of all primers are listed in Supplemental Table 1.

Immunoblot analysis. Total cell extracts or nuclear extracts were separated by SDS-polyacrylamide gels and transferred to PVDF membranes (catalog 162-0177, Bio-Rad). IRE1 phosphorylation was monitored by Phos-tag SDS-PAGE (Wako) as described previously (53). The following antibodies were used for immunoblot analysis: anti-XBP1s (catalog 619502, BioLegend); anti-PERK (catalog 3192, Cell Signaling Technology); anti-IRE1 (catalog 3294, Cell Signaling Technology); anti-ATF6 (catalog BAM-73-500-EX, Cosmo Bio); anti-ATF6 (for mouse Atf6 detection, ref. 54; gift from Ann-Hwee Lee, Weill Cornell Medicine, New York, New York, USA); anti-TBP (catalog ab51841, Abcam); anti-eIF2 $\alpha$ (catalog sc-11386, Santa Cruz Biotechnology Inc.); anti-phospho-eIF2 $\alpha$ (catalog 9721, Cell Signaling Technology); antiMYC (catalog sc-42, Santa Cruz Biotechnology Inc.); anti-GAPDH (catalog sc-32233, Santa Cruz Biotechnology Inc.); anti- $\beta$-actin (cata$\log$ 4970S, Cell Signaling Technology); anti-HA (catalog sc-805, Santa Cruz Biotechnology Inc.); anti-Flag (catalog F7524, MilliporeSigma); anti-MAX (catalog sc-197, Santa Cruz Biotechnology Inc.); and anticaspase-3 (catalog 9662, Cell Signaling Technology).

Luciferase assay. The firefly luciferase reporter for IRE1 promoter or enhancer was constructed by cloning the genomic region of the IRE1 promoter or intron (MYC bound region) into the $M l u \mathrm{I}$ and NcoI sites in pGL3-basic vector (Promega) or the $M l u \mathrm{I}$ and BglII sites in the pGL3-promoter vector (Promega), respectively. All constructs were verified by DNA sequencing. The sequences of all primers are listed in Supplemental Table 1. MYC-responsive 3× E-Box firefly luciferase reporter was a gift from Xin-Hua Feng and its empty control, pBV-Luc, was purchased from Addgene (plasmid 16539). For Figure 3, H and I, and Supplemental Figure 5, A and B, SUM159 cells infected with shScr, shLacZ, shXBP1, or shMYC were cotransfected with 10 ng pRL-PGK (Promega) and 50 ng firefly luciferase reporter plasmids using Lipofectamine 3000. For Figure 4, I-L, BT549 or 293T cells were cotransfected with $10 \mathrm{ng}$ pRL-PGK, 50 ng firefly luciferase reporters, and 50 ng expression plasmids. Cell lysates were collected 48 hours after transfection, and luciferase activities were analyzed using the dualluciferase reporter assay system (catalog E1910, Promega). pRL-PGK, which expresses Renilla luciferase, was used as the internal control for adjustment of discrepancies in transfection and harvest efficiencies.

ChIP assay. SUM159 cells were crosslinked with $1 \%$ formaldehyde for 10 minutes at room temperature. Reaction was quenched with 125 $\mathrm{mM}$ glycine. For PDX tumor, fresh tumor tissues were minced into small pieces on ice and crosslinked with $1.5 \%$ formaldehyde for 10 minutes at room temperature. Reaction was quenched with $125 \mathrm{mM}$ glycine. ChIP was performed as previously described (55) with XBP1 antibody (catalog 619502, BioLegend), MYC antibody (catalog sc-764, Santa Cruz Biotechnology Inc.), P300 antibody (catalog sc-585, Santa Cruz Biotechnology Inc.), SRC3 antibody (catalog 2126, Cell Signaling Technology), CBP 
antibody (catalog sc-583, Santa Cruz Biotechnology Inc.), or normal rabbit IgG control (catalog 2729, Cell Signaling Technology). For ChIP-reChIP assay, MYC or XBP1s antibody was crosslinked to Protein A Sepharose beads (catalog CL-4B, GE Healthcare) using dimethyl pimelimidate dihydrochloride (catalog D8388, MilliporeSigma). The beads were then incubated with chromatin extracts overnight and washed with $0.1 \%$ SDS buffer and eluted with $1 \%$ SDS elution buffer. The eluate was diluted to a final concentration of $0.1 \%$ SDS and incubated with fresh antibodybound Protein A Sepharose beads for the second round of ChIP. The sequences of all primers are listed in Supplemental Table 1.

Coimmunoprecipitation. For exogenous coimmunoprecipitation, $293 \mathrm{~T}$ cells in a 6 -cm dish were transfected with $1 \mu \mathrm{g}$ expression plasmids and harvested 36 hours after transfection. FLAG beads (catalog E2426, MilliporeSigma) or HA beads (catalog E6779, MilliporeSigma) were incubated with the whole cell extracts overnight at $4^{\circ} \mathrm{C}$. For endogenous coimmunoprecipitation, SUM159 or BT549 cells were treated with $5 \mu \mathrm{g} / \mathrm{ml} \mathrm{TM}$ for 6 hours before harvest. Nuclear extracts were incubated with $4 \mu \mathrm{g}$ of anti-XBP1s antibody or anti-MYC antibody (catalog sc-42, Santa Cruz Biotechnology Inc.) overnight at $4^{\circ} \mathrm{C}$. The protein complexes were precipitated by the addition of Protein $\mathrm{A}$ Sepharose beads (catalog CL-4B, GE Healthcare) and incubation for 4 hours at $4^{\circ} \mathrm{C}$. The beads were then washed 5 times, and the precipitated proteins were eluted and analyzed by Western blot.

GST pull-down assay. The GST pull-down assay was performed as previously described (3). Briefly, full-length human XBP1s or MYC was cloned into pET42b (Novagen). The plasmids were transformed into BL21-competent E. coli, resulting in expression of XBP1s or MYC protein. E. coli-expressed proteins were purified via binding to GSH-Sepharose beads (catalog 17075601, GE Healthcare). The protein-bound beads were incubated with XBP1 and MYC full-length or fragment-overexpressed 293T cell lysates overnight at $4^{\circ} \mathrm{C}$. The beads were washed 5 times with cell lysis buffer. The eluents were analyzed by Western blot.

FRET assays. IRE1 endoribonuclease activity was evaluated using fluorescence-based RNA cleavage assays. A stem-loop hairpin RNA substrate (5'-6-FAM-CAUGUCCGCAGCGCAUG-BHQ1-3') mimicking the XBP1 splice site was labeled with 6-FAM and black hole quencher 1 (BHQ1) on its 5' and $3^{\prime}$ terminus, respectively (MilliporeSigma). Cleavage of the stem loop leads to derepression of the FAM signal, which can be measured in real time. Briefly, 8866 compounds were preincubated with $10 \mathrm{nM}$ purified cytoplasmic portions of IRE1 protein or $1 \mathrm{pM}$ RNasA in reaction buffer (50 mM Tris, $\mathrm{pH}$ 7.0, 0.5 $\mathrm{mM} \mathrm{MgCl}_{2}, 10 \mathrm{mM} \mathrm{KCl}, 0.025 \%$ Tween-20, $0.063 \mathrm{mg} / \mathrm{ml}$ tRNA, and $2 \mathrm{mM} \mathrm{DTT}$ ) for 1 hour at room temperature. Upon addition of $100 \mathrm{nM}$ stem-loop RNA substrate (diluted in $10 \mathrm{mM}$ Tris, pH 7.0, 0.1 mM EDTA), cleavage reactions were monitored in real time by fluorescence intensity at 1-minute intervals using the QuantStudio 6 Flex Real-Time PCR System (Thermo Fisher Scientific). No enzyme and no substrate groups served as negative controls.

Gel-based RNase L in vitro cleavage assay. Full-length RNase $\mathrm{L}$ at $0.4 \mathrm{nM}$ was activated with $5 \mathrm{nM} 2^{\prime}-5$ 'oligoadenylate (2-5A) and $50 \mu \mathrm{M}$ ADP. The activated RNase $\mathrm{L}$ was incubated with 8866 ranging from $0.5 \mathrm{nM}$ to $50,000 \mathrm{nM}$ for 30 minutes on ice, and then $135 \mathrm{nM}$ of the 6-FAM-RNA-BHQ1 substrate (5'-6-FAMUUAUCAAAUUCUUAUUUGCCCCAUUUUUUUGGUUUABHQ1-3') was added to the mixture. Time points were taken at 5, 10, and 20 minutes, and the samples were run on a UREA-TBE RNA gel. The gel fluorescence was read at $488 \mathrm{~nm}$ using a GE Healthcare Typhoon Variable Mode Imager.
Murine and yeast IRE1 in vitro cleavage assay. For murine (mIre1) and yeast IRE1 (scIRE1) in vitro cleavage assay, murine and yeast fulllength IRE1 at $100 \mathrm{nM}$ and $1 \mu \mathrm{M}$, respectively, with the latter activated with $2.5 \mathrm{mM}$ ADP, were incubated with 8866 ranging from 0.00056 $\mu \mathrm{M}$ to $100 \mu \mathrm{M}$ for 30 minutes on ice. On addition of the stem-loop hairpin XBP1 RNA substrate (5'-Alexa Fluor 647-CAUGUCCGCAGCGCAUG-BHQ1-3') at $100 \mathrm{nM}$ and $25 \mathrm{nM}$ for murine and yeast IRE1, respectively, cleavage reactions were monitored in real time by fluorescence intensity ( $\lambda$ excited $=651 \mathrm{~nm}, \lambda$ emission $=672 \mathrm{~nm}$ ) at 2 -minute intervals using a Molecular Dimensions Analyst HT microplate reader.

Determination of $E C_{50}$. WT or IRE1-KO SUM159 cells were seeded into 6-well plates at 500 cells per well and treated with DMSO or various doses of 8866 for 10 days. Colonies were fixed in methanol, stained with crystal violet (0.5\% crystal violet, $20 \%$ methanol), and counted. The percentage of response was calculated as $\left(N_{D}-N_{8}\right) / N_{D} \times 100 \%$, where $N_{D}$ is the colony number of DMSO-treated cells and $N_{8}$ is the colony number of 8866-treated cells. Concentration-response curves were plotted, and the $x$ values were transformed to log form. The $\mathrm{EC}_{50}$ values were estimated using nonlinear regression analysis (56).

In vivo dose test of 8866.8866 was provided by Fosun Orinove Inc. The vehicle for 8866 is $1 \%$ microcrystalline cellulose in $50 \%$ sucrose. 8866 Formulation was homogenized in a water bath sonicator for 1 hour before dosing. Five- to six-week-old C57BL/6 mice from Envigo were treated with either vehicle or 8866 through 1-time oral gavage. All mice received $1 \mathrm{mg} / \mathrm{kg}$ TM (first dissolved in DMSO and then diluted in $150 \mathrm{mM}$ dextrose) through i.p. injection 6 hours prior to sacrifice. Mouse livers were harvested 24 hours after oral gavage, and Xbp1 splicing was tested using reverse-transcriptase PCR (RT-PCR).

Tumor inoculation and treatment. The establishment of human PDX and p53-null GEM tumor lines was described in detail previously (37). Fresh xenograft tumor fragments were transplanted into the cleared mammary fat pads of 3- to 4-week-old female SCID/beige mice (from Envigo, for human PDX tumor) or BALB/c mice (from Envigo, for p53-null GEM tumor). When tumors reached a volume of approximately $150-350 \mathrm{~mm}^{3}$, mice were randomized and treated with drugs. The 8866 was administered via daily oral gavage at $300 \mathrm{mg} /$ $\mathrm{kg}$ dose. Docetaxel (MilliporeSigma) was dissolved in 25\% Tween 80 and $12 \%$ ethanol and administered through weekly i.p. injections at 10 $\mathrm{mg} / \mathrm{kg}$ dose. CHIR99021 (catalog C-6556, LC Lab) was suspended in $4 \%$ DMSO, 10\% PEG400, and 10\% Tween 80 and administered through i.p. injection every 2 days at $30 \mathrm{mg} / \mathrm{kg}$ dose. Mice undergoing monotherapy also received vehicle in the combination treatment study. The ethical end point for tumor transplantation experiments was reached when a tumor reached $1.5 \mathrm{~cm}$ or more in any dimension. Tumor volumes were monitored using calipers every 5 days and calculated according to the formula $l \times w^{2} / 2$, where $l$ represents tumor length and $w$ represents tumor width. For in vivo BrdU labeling of tumors, mice were i.p. injected with BrdU-labeling reagent (catalog 00-0103, Invitrogen) at a dose of $10 \mu \mathrm{l} / \mathrm{g}$ body weight. Tumors were harvested 2 hours after injection.

For lung metastasis experiments, 200,000 luciferase-labeled MDA-MB-231-LM2 cells were injected into 5- to 6-week-old female athymic nude mice (Envigo) through the tail vein. Two days later, mice were randomized and treated with vehicle, $300 \mathrm{mg} / \mathrm{kg} 8866$ (daily oral gavage), docetaxel (10 mg/kg, weekly i.p. injection), or the combination of 8866 and docetaxel. Lung metastasis was measured by bioluminescence imaging using IVIS Lumina II equipment. 
Tumor immunophenotyping. Fresh tumor tissues were chopped into small pieces, followed by digestion in RPMI containing $0.25 \mathrm{mg} / \mathrm{ml}$ Liberase TL (catalog 05401020001, Roche) and $0.2 \mathrm{mg} / \mathrm{ml}$ DNase I (catalog 10104159001, Roche) for 1 hour at $37^{\circ} \mathrm{C}$. Digestion was terminated by adding RPMI containing $10 \% \mathrm{FBS}$, and single cells were filtered through a $40-\mu \mathrm{m}$ cell restrainer. Cells were washed with PBS and incubated with Fc blocker (catalog 101302, BioLegend) on ice for 10 minutes. Cell suspension was then stained with anti-CD45 PE (catalog 103105), anti-CD45 FITC (catalog 103108), anti-F4/80 PerCP-Cy5.5 (catalog 123127), anti-CD11b PE-Cy7 (catalog 101215), anti-CD11c APC-Cy7 (catalog 117323), anti-Gr1 FITC (catalog 108405), anti-B220 PE (catalog 103207), anti-CD3 PE-Cy7 (catalog 100320), anti-CD8 PerCP-Сy5.5 (catalog 100734), anti-CD4 APC-Cy7 (catalog 100414), and DAPI (cata$\log$ 422801) antibodies from BioLegend. Samples were run on Attune NxT Flow Cytometer, and data were analyzed using FlowJo software.

Histology, IHC, and TUNEL staining. Tissue specimens were fixed in fresh $4 \%$ paraformaldehyde for 24 hours and stored in $70 \%$ ethanol until paraffin embedding. Breast carcinoma tissue microarray (catalog BR1201) and human normal mammary gland tissue sections (catalog HuFPT127) were purchased from Biomax. H\&E and IHC staining were performed on $5 \mu \mathrm{m}$-thick paraffin sections. The following primary antibodies were used in IHC: MYC (1:300, catalog ab32072, Abcam), IRE1 (1:200, catalog 3294, Cell Signaling Technology), BrdU (1:250, catalog ab6326, Abcam), CD31 (1:50, catalog ab28364, Abcam), and cleaved caspase-3 (1:200, catalog 9664, Cell Signaling Technology). Slides were incubated with biotinylated secondary antibody and ABC-HRP (both from Vector Laboratories). Sections were developed with DAB and counterstained with hematoxylin. For quantifications of TMA staining, TMAs were scanned using the Aperio scanner. MYC expression was evaluated at $\times 200$ magnification. For each core, 3 images of representative areas were analyzed and a total of 1,000 to 2,000 tumor cells were counted. IHC scoring was performed using Histoscore (H-score) calculated by the Aperio IHC nuclear image analysis system, which included a semiquantitative assessment of both fraction of positive cells and intensity of staining (57). The intensity score was defined as no staining (score 0), weak (score 1), moderate (score 2), or strong (score 3) staining. The fraction score was based on the proportion of positively stained cells $(0 \%-100 \%)$. The intensity and fraction scores were then multiplied to obtain the $\mathrm{H}$-score, which ranged from 0 to 3 and represented the level of MYC. IRE1 expression was evaluated per the intensity of staining. The intensity was defined as no staining (score 0), weak (score 1), moderate (score 2), or strong (score 3) staining. Intensity with 0 and 1 was classified as low intensity; intensity with 2 was classified as medium intensity; and intensity with 3 was classified as high intensity. Images with $\times 100$ magnification were scored as the total number of positively stained cells per captured field for the quantitation of cleaved caspase-3. Any discrete cluster or single cell with positive CD31 staining was counted as 1 microvessel. All quantification was carried out in a manner that was blinded to the treatment group.

TUNEL staining was performed on paraffin-embedded tissue sections using the In Situ Cell Death Detection Kit (catalog 11684795910, Roche) following the manufacturer's instructions. Sections were counterstained with DAPI, and images were captured under fluorescence microscope. Tissue sections incubated with TUNEL reaction buffer without dTd enzyme served as negative controls. Tissue sections treated with DNase I served as positive controls.

Statistics. Data showing tumor volume, mouse body weight, immunophenotyping, and metastasis quantification are presented as the mean \pm SEM. Data from all other experiments are presented as the mean \pm SD. Student's $t$ test was utilized to compare the differences between 2 groups. One-way ANOVA with Tukey's multiple comparison test was used to compare the differences among 3 or more groups. Twoway ANOVA with Bonferroni's post test was used to calculate the significance difference for tumor volume and body weight measurement over time. The log-rank test was used to test for the significant differences of survival between the groups. The extra sum-of-squares $\mathrm{F}$ test was used to calculate the significant difference in $\mathrm{EC}_{50}$ of 8866 between groups. Analyses were performed with GraphPad Prism, version 5 (GraphPad Software Inc.). $P<0.05$ was considered statistically significant.

Study approval. All protocols described in this study were approved by the Baylor College of Medicine Institutional Animal Care and Use Committee.

\section{Author contributions}

NZ, JC, LX, and XC developed the hypothesis, designed experiments, and analyzed the data. NZ, JC, LX, QT, LED, XDL, MT, YL, XW, DZH, QS, YX, YW, X Liu, WB, YJ, ML, YG, ZS, HY, BY, X Lin, XHF, SMH, FL, HS, YC, LH, BAK, NP, FS, JMR, MTL, and XC performed experiments and contributed to experimental design and generation of the reagents. JMR, MTL, NZ, JC, and LX contributed to manuscript editing. MTL, JMR, QZ, and JBP provided key reagents. XC conceived the project, supervised the work, and wrote the manuscript.

\section{Acknowledgments}

We thank Qin Yan (Yale University) for providing the iCas9 system. We are grateful to Yi Li, Xiang Zhang, and Thomas Westbrook (Baylor College of Medicine) for advice and discussions. This work was supported by the National Cancer Institute (NCI) (5P50CA186784-03 SPORE Career Development Award to XC and SPORE Supplement Award to MTL and LED), the Cancer Prevention and Research Institutes of Texas (CPRIT) (RR150009 to XC; RR140071 to YC), Susan G. Komen for the Cure (CCR16380871 to XC), the NIH (R01CA148761 to JMR; R00CA175290 to YC; R01DK114356 and R03DK105006 to $\mathrm{SMH}$ ), the Cytometry and Cell Sorting Core (funded by NIH P30 AI036211,P30 CA125123, and S10 RR024574), and PDX and Advance In Vivo Models Core at Baylor College of Medicine (funding from RP170691 CPRIT Core Facility Support Award and NCI-CA125123 P30 Cancer Center Support Grant).

Address correspondence to: Xi Chen, Department of Molecular and Cellular Biology, One Baylor Plaza, MS: BCM130, Debakey Building, BCMM-M626, Houston, Texas 77030, USA. Phone: 713.798.4398; Email: Xi.Chen@bcm.edu.
1. Wang M, Kaufman RJ. The impact of the endoplasmic reticulum protein-folding environment on cancer development. Nat Rev Cancer. 2014;14(9):581-597.
2. Clarke HJ, Chambers JE, Liniker E, Marciniak SJ. Endoplasmic reticulum stress in malignancy. Cancer Cell. 2014;25(5):563-573.

3. Chen X, et al. XBP1 promotes triple-negative breast cancer by controlling the HIF1 $\alpha$ pathway. Nature. 2014;508(7494):103-107.

4. Feng YX, et al. Epithelial-to-mesenchymal transition activates PERK-eIF2 $\alpha$ and sensitizes cells 
to endoplasmic reticulum stress. Cancer Discov. 2014;4(6):702-715.

5. Mimura N, et al. Blockade of XBP1 splicing by inhibition of IRE1 $\alpha$ is a promising therapeutic option in multiple myeloma. Blood. 2012;119(24):5772-5781.

6. Cubillos-Ruiz JR, et al. ER stress sensor XBP1 controls anti-tumor immunity by disrupting dendritic cell homeostasis. Cell. 2015;161(7):1527-1538.

7. Condamine T, et al. ER stress regulates myeloid-derived suppressor cell fate through TRAIL-R-mediated apoptosis. J Clin Invest. 2014;124(6):2626-2639.

8. Walter P, Ron D. The unfolded protein response: from stress pathway to homeostatic regulation. Science. 2011;334(6059):1081-1086.

9. Ron D, Walter P. Signal integration in the endoplasmic reticulum unfolded protein response. Nat Rev Mol Cell Biol. 2007;8(7):519-529.

10. Cox JS, Shamu CE, Walter P. Transcriptional induction of genes encoding endoplasmic reticulum resident proteins requires a transmembrane protein kinase. Cell.1993;73(6):1197-1206.

11. Sidrauski C, Walter P. The transmembrane kinase Ire1p is a site-specific endonuclease that initiates mRNA splicing in the unfolded protein response. Cell.1997;90(6):1031-1039.

12. Mori K, Ma W, Gething MJ, Sambrook J. A transmembrane protein with a cdc2+/CDC28-related kinase activity is required for signaling from the ER to the nucleus. Cell. 1993;74(4):743-756.

13. Korennykh AV, et al. The unfolded protein response signals through high-order assembly of Ire1. Nature. 2009;457(7230):687-693.

14. Lee KP, Dey M, Neculai D, Cao C, Dever TE, Sicheri F. Structure of the dual enzyme Ire1 reveals the basis for catalysis and regulation in nonconventional RNA splicing. Cell. 2008;132(1):89-100.

15. Yoshida H, Matsui T, Yamamoto A, Okada T, Mori K. XBP1 mRNA is induced by ATF 6 and spliced by IRE1 in response to ER stress to produce a highly active transcription factor. Cell. 2001;107(7):881-891.

16. Shen $X$, et al. Complementary signaling pathways regulate the unfolded protein response and are required for C. elegans development. Cell. 2001;107(7):893-903.

17. Calfon M, et al. IRE1 couples endoplasmic reticulum load to secretory capacity by processing the XBP-1 mRNA. Nature. 2002;415(6867):92-96.

18. Cross BC, et al. The molecular basis for selective inhibition of unconventional mRNA splicing by an IRE1-binding small molecule. Proc Natl Acad Sci USA. 2012;109(15):E869-E878.

19. Papandreou I, et al. Identification of an Ire1alpha endonuclease specific inhibitor with cytotoxic activity against human multiple myeloma. Blood. 2011;117(4):1311-1314.

20. Atkins $C$, et al. Characterization of a novel PERK kinase inhibitor with antitumor and antiangiogenic activity. Cancer Res. 2013;73(6):1993-2002.

21. Sidrauski C, et al. Pharmacological brake-release of mRNA translation enhances cognitive memo- ry. Elife. 2013;2:e00498.

22. Gallagher CM, et al. Ceapins are a new class of unfolded protein response inhibitors, selectively targeting the ATF6 $\alpha$ branch. Elife. 2016;5:e11878.

23. Han D, et al. IRE1alpha kinase activation modes control alternate endoribonuclease outputs to determine divergent cell fates. Cell. 2009;138(3):562-575.

24. Maly DJ, Papa FR. Druggable sensors of the unfolded protein response. Nat Chem Biol. 2014;10(11):892-901.

25. Cancer Genome Atlas Network. Comprehensive molecular portraits of human breast tumours. Nature. 2012;490(7418):61-70.

26. Horiuchi D, et al. MYC pathway activation in triple-negative breast cancer is synthetic lethal with CDK inhibition.JExp Med. 2012;209(4):679-696.

27. Kessler JD, et al. A SUMOylation-dependent transcriptional subprogram is required for Myc-driven tumorigenesis. Science. 2012;335(6066):348-353.

28. Kugel S, et al. SIRT6 suppresses pancreatic cancer through control of Lin28b. Cell. 2016;165(6):1401-1415.

29. Lin CY, et al. Transcriptional amplification in tumor cells with elevated c-Myc. Cell. 2012;151(1):56-67.

30. Dang CV. MYC on the path to cancer. Cell. 2012;149(1):22-35.

31. Grandori C, Cowley SM, James LP, Eisenman RN. The Myc/Max/Mad network and the transcriptional control of cell behavior. Annu Rev Cell Dev Biol. 2000;16:653-699.

32. Yamamoto K, Yoshida H, Kokame K, Kaufman RJ, Mori K. Differential contributions of ATF6 and XBP1 to the activation of endoplasmic reticulum stress-responsive cis-acting elements ERSE, UPRE and ERSE-II. J Biochem. 2004;136(3):343-350.

33. Sanches M, et al. Structure and mechanism of action of the hydroxy-aryl-aldehyde class of IRE1 endoribonuclease inhibitors. Nat Commun. 2014;5:4202.

34. Huang $\mathrm{H}$, et al. Dimeric structure of pseudokinase RNase $\mathrm{L}$ bound to 2-5A reveals a basis for interferon-induced antiviral activity. Mol Cell. 2014;53(2):221-234.

35. Zhou A, Hassel BA, Silverman RH. Expression cloning of 2-5A-dependent RNAase: a uniquely regulated mediator of interferon action. Cell. 1993;72(5):753-765

36. Hollien J, Weissman JS. Decay of endoplasmic reticulum-localized mRNAs during the unfolded protein response. Science. 2006;313(5783):104-107.

37. Zhang $\mathrm{X}$, et al. A renewable tissue resource of phenotypically stable, biologically and ethnically diverse, patient-derived human breast cancer xenograft models. Cancer Res. 2013;73(15):4885-4897.

38. Yada M, et al. Phosphorylation-dependent degradation of c-Myc is mediated by the F-box protein Fbw7. EMBO J. 2004;23(10):2116-2125.

39. Welcker M, et al. The Fbw7 tumor suppressor regulates glycogen synthase kinase 3 phosphorylation-dependent c-Myc protein degradation. Proc Natl Acad Sci USA. 2004;101(24):9085-9090. 40. Bechard M, Dalton S. Subcellular localization of glycogen synthase kinase 3beta controls embryonic stem cell self-renewal. Mol Cell Biol. 2009;29(8):2092-2104.

41. Carey L, Winer E, Viale G, Cameron D, Gianni L. Triple-negative breast cancer: disease entity or title of convenience? Nat Rev Clin Oncol. 2010;7(12):683-692.

42. Lee AH, Scapa EF, Cohen DE, Glimcher LH. Regulation of hepatic lipogenesis by the transcription factor XBP1. Science. 2008;320(5882):1492-1496.

43. Howard SC, Jones DP, Pui CH. The tumor lysis syndrome. N Engl JMed. 2011;364(19):1844-1854

44. Herschkowitz JI, et al. Comparative oncogenomics identifies breast tumors enriched in functional tumor-initiating cells. Proc Natl Acad Sci USA. 2012;109(8):2778-2783.

45. Jerry DJ, et al. A mammary-specific model demonstrates the role of the p53 tumor suppressor gene in tumor development. Oncogene. 2000;19(8):1052-1058.

46. Pfefferle AD, et al. Transcriptomic classification of genetically engineered mouse models of breast cancer identifies human subtype counterparts. Genome Biol. 2013;14(11):R125.

47. Chen DS, Mellman I. Elements of cancer immunity and the cancer-immune set point. Nature. 2017;541(7637):321-330.

48. Luo J, Solimini NL, Elledge SJ. Principles of cancer therapy: oncogene and non-oncogene addiction. Cell. 2009;136(5):823-837.

49. Hart LS, et al. ER stress-mediated autophagy promotes Myc-dependent transformation and tumor growth. J Clin Invest. 2012;122(12):4621-4634.

50. Xie H, et al. IRE1 $\alpha$ RNase-dependent lipid homeostasis promotes survival in Myc-transformed cancers. JClin Invest. 2018;128(4):1300-1316.

51. Tang $\mathrm{CH}$, et al. Inhibition of ER stress-associated IRE-1/XBP-1 pathway reduces leukemic cell survival. JClin Invest. 2014;124(6):2585-2598.

52. Cao J, et al. An easy and efficient inducible CRISPR/Cas9 platform with improved specificity for multiple gene targeting. Nucleic Acids Res. 2016;44(19):e149.

53. Yang L, Xue Z, He Y, Sun S, Chen H, Qi L. A Phostag-based approach reveals the extent of physiological endoplasmic reticulum stress. PLoS One. 2010;5(7):e11621.

54. Fedeles SV, et al. Sec63 and Xbp1 regulate IRE1a activity and polycystic disease severity. JClin Invest. 2015;125(5):1955-1967.

55. Chen $X$, et al. Integration of external signaling pathways with the core transcriptional network in embryonic stem cells. Cell. 2008;133(6):1106-1117.

56. Lyles RH, Poindexter C, Evans A, Brown M, Cooper CR. Nonlinear model-based estimates of IC(50) for studies involving continuous therapeutic dose-response data. Contemp Clin Trials. 2008;29(6):878-886.

57. Akbar S, Jordan LB, Purdie CA, Thompson AM, McKenna SJ. Comparing computergenerated and pathologist-generated tumour segmentations for immunohistochemical scoring of breast tissue microarrays. Br JCancer. 2015;113(7):1075-1080. 